\title{
A $q$-Analogue of the Centralizer Construction and Skew Representations of the Quantum Affine Algebra
}

\author{
Mark J. HOPKINS and Alexander I. MOLEV ${ }^{*}$ \\ School of Mathematics and Statistics, University of Sydney, NSW 2006, Australia \\ E-mail: markh@maths.usyd.edu.au,alexm@maths.usyd.edu.au \\ * URL: http://www.maths.usyd.edu.au/u/alexm
}

Received October 14, 2006; Published online December 26, 2006

Original article is available at http://www.emis.de/journals/SIGMA/2006/Paper092/

\begin{abstract}
We prove an analogue of the Sylvester theorem for the generator matrices of the quantum affine algebra $\mathrm{U}_{q}\left(\widehat{\mathfrak{g l}}_{n}\right)$. We then use it to give an explicit realization of the skew representations of the quantum affine algebra. This allows one to identify them in a simple way by calculating their highest weight, Drinfeld polynomials and the Gelfand-Tsetlin character (or $q$-character). We also apply the quantum Sylvester theorem to construct a $q$-analogue of the Olshanski algebra as a projective limit of certain centralizers in $\mathrm{U}_{q}\left(\mathfrak{g l}_{n}\right)$ and show that this limit algebra contains the $q$-Yangian as a subalgebra.
\end{abstract}

Key words: quantum affine algebra; quantum Sylvester theorem; skew representations

2000 Mathematics Subject Classification: 81R10

To the memory of Vadim Kuznetsov

\section{Introduction}

The quantum affine algebras form an important family of quantized enveloping algebras. They were introduced by Drinfeld and Jimbo in 1985 in the general context of quantum groups and have since been studied by many authors; see e.g. the books by Chari and Pressley [4] or Klimyk and Schmüdgen [13] for a detailed account of the origins and applications of the quantum group theory. A particular RTT-presentation of the quantum affine algebras and their rational counterparts (now known as the Yangians, due to Drinfeld) was originally introduced and studied in the work of L.D. Faddeev and the Leningrad school of mathematical physicists in the late 70's and early 80's in relation with the quantum inverse scattering method (QISM); see e.g. Reshetikhin, Takhtajan and Faddeev [30] for the basics of this approach. The representation theory of the quantum affine algebras and Yangians, as well as various applications of the theory, are discussed in the mentioned above book [4, Chapter 12]. Amongst other applications we note a surprising connection of the QISM I and QISM II algebras with the hypergeometric functions discovered by Kuznetsov and Koornwinder [16, 17].

By the results of Olshanski $[27,28]$, the $A$ type Yangian is recovered as a certain projective limit of centralizers in the universal enveloping algebras. In this paper, we develop a $q$-analogue of the Olshanski construction where the role of the Yangian is played by the quantum affine algebra. Let us now discuss this approach in more detail.

\footnotetext{
${ }^{\star}$ This paper is a contribution to the Vadim Kuznetsov Memorial Issue "Integrable Systems and Related Topics". The full collection is available at http://www.emis.de/journals/SIGMA/kuznetsov.html
} 
Let $n$ and $m$ be integers such that $n>m \geqslant 0$. Denote by $\mathrm{A}_{m}(n)$ the centralizer of $\mathfrak{g l}_{n-m}$ in the universal enveloping algebra $\mathrm{U}\left(\mathfrak{g l}_{n}\right)$, where $\mathfrak{g l}_{n-m}$ is regarded as a natural subalgebra of $\mathfrak{g l}_{n}$. Due to Olshanski $[27,28]$, for any fixed $m$ there exists a chain of natural homomorphisms

$$
\mathrm{A}_{m}(m) \leftarrow \mathrm{A}_{m}(m+1) \leftarrow \cdots \leftarrow \mathrm{A}_{m}(n) \leftarrow \cdots
$$

which respect the natural filtrations inherited from the universal enveloping algebra. So, one can define the corresponding projective limit algebra

$$
\mathrm{A}_{m}=\lim \operatorname{proj} \mathrm{A}_{m}(n), \quad n \rightarrow \infty
$$

in the category of filtered algebras. In particular, the algebra $\mathrm{A}_{0}$ is the projective limit of the centers of $\mathrm{U}\left(\mathfrak{g l}_{n}\right)$ and it is isomorphic to an algebra of polynomials in countably many variables. Moreover, for $m>0$ one has the isomorphism [27, 28]:

$$
\mathrm{A}_{m} \cong \mathrm{A}_{0} \otimes \mathrm{Y}\left(\mathfrak{g l}_{m}\right)
$$

where $\mathrm{Y}\left(\mathfrak{g l}_{m}\right)$ is the Yangian for the Lie algebra $\mathfrak{g l}_{m}$. The algebra $\mathrm{Y}\left(\mathfrak{g l}_{m}\right)$ is a deformation of the enveloping algebra $\mathrm{U}\left(\mathfrak{g l}_{m} \otimes \mathbb{C}[x]\right)$ in the class of Hopf algebras; see Drinfeld [7]. A key part of the proof of the decomposition (1.3) is a construction of algebra homomorphisms

$$
\mathrm{Y}\left(\mathfrak{g l}_{m}\right) \rightarrow \mathrm{A}_{m}(n), \quad n=m, m+1, \ldots
$$

compatible with the chain (1.1) which define an embedding

$$
\mathrm{Y}\left(\mathfrak{g l}_{m}\right) \hookrightarrow \mathrm{A}_{m}
$$

A similar construction can be applied to the orthogonal and symplectic Lie algebras which leads to the introduction of the corresponding twisted Yangians; see Olshanski [29] and Molev and Olshanski [21]. A super analogue of the centralizer construction was given by Nazarov [24] to obtain the Yangian for the queer Lie superalgebra. Moreover, the homomorphism (1.4) gives rise to a representation of the Yangian in the homomorphism space $\operatorname{Hom}_{\mathfrak{g l}_{m}}(L(\mu), L(\lambda))$ which is called the skew (or elementary) representation. Such representations were studied by Cherednik [5] (mainly in the context of the quantum affine algebras) and by Nazarov and Tarasov [26]; see also [19].

In this paper we give a $q$-analogue of the centralizer construction where $\mathrm{U}\left(\mathfrak{g l}_{n}\right)$ is replaced by the quantized enveloping algebra $\mathrm{U}_{q}\left(\mathfrak{g l}_{n}\right)$. We follow the approach of [19] where an alternative embedding (1.5) was constructed. It is based upon a version of the quantum Sylvester theorem (see Theorem 3.6 below); cf. Gelfand and Retakh [10], Krob and Leclerc [15]. Here the Yangian $\mathrm{Y}\left(\mathfrak{g l}_{m}\right)$ should be replaced with the $q$-Yangian $\mathrm{Y}_{q}\left(\mathfrak{g l}_{m}\right)$ which is a natural subalgebra of the quantum affine algebra $\mathrm{U}_{q}\left(\widehat{\mathfrak{g l}}_{m}\right)$. We prove that the $q$-Yangian is embedded into an appropriate $q$-analogue of the Olshanski algebra $\mathrm{A}_{m}$ (see Theorem 5.4 below). In particular, this gives a proof of the Poincaré-Birkhoff-Witt theorem for the $q$-Yangian, and the same method applies to provide a new proof of this theorem for the Yangian $\mathrm{Y}\left(\mathfrak{g l}_{m}\right)$. We believe that a $q$-version of the isomorphism (1.3) also takes place, however, we do not have a proof.

Using a homomorphism associated with the quantum Sylvester theorem, we provide an explicit construction of the skew representations of the quantum affine algebra $\mathrm{U}_{q}\left(\widehat{\mathfrak{g l}}_{n}\right)$. We generally follow the approach of [19], simplifying the arguments with the use of some observations of Brundan and Kleshchev [2]. The construction allows us to easily identify the skew representations by calculating their highest weights, Drinfeld polynomials and the GelfandTsetlin characters. In the Yangian case, the Drinfeld polynomials were calculated by Nazarov and Tarasov [26]; see also [1, 5, 23]. In a more general context, the Gelfand-Tsetlin characters were introduced by Brundan and Kleshchev [3] in their study of representations of the shifted Yangians. They are analogous to the Yangian characters of Knight [14] and the $q$-characters of Frenkel and Reshetikhin [9] for the quantum affine algebras. 


\section{Definitions and preliminaries}

We shall use an $R$-matrix presentation of the algebra $\mathrm{U}_{q}\left(\mathfrak{g l}_{n}\right)$ following [11]; see also [13] for more details. We fix a complex parameter $q$ which is nonzero and not a root of unity. Consider the $R$-matrix

$$
R=q \sum_{i} E_{i i} \otimes E_{i i}+\sum_{i \neq j} E_{i i} \otimes E_{j j}+\left(q-q^{-1}\right) \sum_{i<j} E_{i j} \otimes E_{j i}
$$

which is an element of End $\mathbb{C}^{n} \otimes$ End $\mathbb{C}^{n}$, where the $E_{i j}$ denote the standard matrix units and the indices run over the set $\{1, \ldots, n\}$. The $R$-matrix satisfies the Yang-Baxter equation

$$
R_{12} R_{13} R_{23}=R_{23} R_{13} R_{12},
$$

where both sides take values in End $\mathbb{C}^{n} \otimes$ End $\mathbb{C}^{n} \otimes$ End $\mathbb{C}^{n}$ and the indices indicate the copies of End $\mathbb{C}^{n}$, e.g., $R_{12}=R \otimes 1$ etc.

The quantized enveloping algebra $\mathrm{U}_{q}\left(\mathfrak{g l}_{n}\right)$ is generated by elements $t_{i j}$ and $\bar{t}_{i j}$ with $1 \leqslant i, j \leqslant n$ subject to the relations

$$
\begin{aligned}
& t_{i j}=\bar{t}_{j i}=0, \quad 1 \leqslant i<j \leqslant n, \\
& t_{i i} \bar{t}_{i i}=\bar{t}_{i i} t_{i i}=1, \quad 1 \leqslant i \leqslant n, \\
& R T_{1} T_{2}=T_{2} T_{1} R, \quad R \bar{T}_{1} \bar{T}_{2}=\bar{T}_{2} \bar{T}_{1} R, \quad R \bar{T}_{1} T_{2}=T_{2} \bar{T}_{1} R .
\end{aligned}
$$

Here $T$ and $\bar{T}$ are the matrices

$$
T=\sum_{i, j} t_{i j} \otimes E_{i j}, \quad \bar{T}=\sum_{i, j} \bar{t}_{i j} \otimes E_{i j},
$$

which are regarded as elements of the algebra $\mathrm{U}_{q}\left(\mathfrak{g l}_{n}\right) \otimes$ End $\mathbb{C}^{n}$. Both sides of each of the $R$ matrix relations in (2.3) are elements of $\mathrm{U}_{q}\left(\mathfrak{g l}_{n}\right) \otimes$ End $\mathbb{C}^{n} \otimes$ End $\mathbb{C}^{n}$ and the indices of $T$ and $\bar{T}$ indicate the copies of End $\mathbb{C}^{n}$ where $T$ or $\bar{T}$ acts; e.g. $T_{1}=T \otimes 1$. In terms of the generators the defining relations between the $t_{i j}$ can be written as

$$
q^{\delta_{i j}} t_{i a} t_{j b}-q^{\delta_{a b}} t_{j b} t_{i a}=\left(q-q^{-1}\right)\left(\delta_{b<a}-\delta_{i<j}\right) t_{j a} t_{i b}
$$

where $\delta_{i<j}$ equals 1 if $i<j$ and 0 otherwise. The relations between the $\bar{t}_{i j}$ are obtained by replacing $t_{i j}$ by $\bar{t}_{i j}$ everywhere in (2.5). Finally, the relations involving both $t_{i j}$ and $\bar{t}_{i j}$ have the form

$$
q^{\delta_{i j}} \bar{t}_{i a} t_{j b}-q^{\delta_{a b}} t_{j b} \bar{t}_{i a}=\left(q-q^{-1}\right)\left(\delta_{b<a} t_{j a} \bar{t}_{i b}-\delta_{i<j} \bar{t}_{j a} t_{i b}\right) .
$$

It is well known that the algebra $\mathrm{U}_{q}\left(\mathfrak{g l}_{n}\right)$ specializes to $\mathrm{U}\left(\mathfrak{g l}_{n}\right)$ as $q \rightarrow 1$. To make this more precise, regard $q$ as a formal variable and $\mathrm{U}_{q}\left(\mathfrak{g l}_{n}\right)$ as an algebra over $\mathbb{C}(q)$. Then set $\mathcal{A}=\mathbb{C}\left[q, q^{-1}\right]$ and consider the $\mathcal{A}$-subalgebra $\mathrm{U}_{\mathcal{A}}$ of $\mathrm{U}_{q}\left(\mathfrak{g l}_{n}\right)$ generated by the elements

$$
\frac{t_{i j}}{q-q^{-1}} \quad \text { for } \quad i>j, \quad \frac{\bar{t}_{i j}}{q-q^{-1}} \quad \text { for } \quad i<j
$$

and

$$
\frac{t_{i i}-1}{q-1}, \quad \frac{\bar{t}_{i i}-1}{q-1},
$$

for $i=1, \ldots, n$. Then we have an isomorphism

$$
\mathrm{U}_{\mathcal{A}} \otimes_{\mathcal{A}} \mathbb{C} \cong \mathrm{U}\left(\mathfrak{g l}_{n}\right)
$$


with the action of $\mathcal{A}$ on $\mathbb{C}$ defined via the evaluation $q=1$; see e.g. [4, Section 9.2]. Note that the elements (2.7) respectively specialize to the elements $E_{i j}$ and $-E_{i j}$ of $\mathrm{U}\left(\mathfrak{g l}_{n}\right)$ while the elements (2.8) specialize to $E_{i i}$ and $-E_{i i}$.

The quantized enveloping algebra $\mathrm{U}_{q}\left(\mathfrak{s l}_{n}\right)$ is defined as the associative algebra with generators $k_{1}, \ldots, k_{n-1}, k_{1}^{-1}, \ldots, k_{n-1}^{-1}, e_{1}, \ldots, e_{n-1}$ and $f_{1}, \ldots, f_{n-1}$ subject to the defining relations

$$
\begin{aligned}
& k_{i} k_{j}=k_{j} k_{i}, \quad k_{i} k_{i}^{-1}=k_{i}^{-1} k_{i}=1, \\
& k_{i} e_{j} k_{i}^{-1}=q^{a_{i j}} e_{j}, \quad k_{i} f_{j} k_{i}^{-1}=q^{-a_{i j}} f_{j}, \\
& {\left[e_{i}, f_{j}\right]=\delta_{i j} \frac{k_{i}-k_{i}^{-1}}{q-q^{-1}},} \\
& {\left[e_{i}, e_{j}\right]=\left[f_{i}, f_{j}\right]=0 \quad \text { if }|i-j|>1,} \\
& e_{i}^{2} e_{j}-\left(q+q^{-1}\right) e_{i} e_{j} e_{i}+e_{j} e_{i}^{2}=0 \quad \text { if }|i-j|=1, \\
& f_{i}^{2} f_{j}-\left(q+q^{-1}\right) f_{i} f_{j} f_{i}+f_{j} f_{i}^{2}=0 \quad \text { if }|i-j|=1,
\end{aligned}
$$

where $\left[a_{i j}\right]$ denotes the Cartan matrix associated with the Lie algebra $\mathfrak{s l}_{n}$ so that its only nonzero entries are $a_{i i}=2$ and $a_{i j}=-1$ for $|i-j|=1$.

We have an embedding $\mathrm{U}_{q}\left(\mathfrak{s l}_{n}\right) \hookrightarrow \mathrm{U}_{q}\left(\mathfrak{g l}_{n}\right)$ given by

$$
\begin{aligned}
k_{i} & \mapsto t_{i i} \bar{t}_{i+1, i+1}, & k_{i}^{-1} \mapsto \bar{t}_{i i} t_{i+1, i+1}, \\
e_{i} & \mapsto-\frac{\bar{t}_{i, i+1} t_{i i}}{q-q^{-1}}, & f_{i} \mapsto \frac{\bar{t}_{i i} t_{i+1, i}}{q-q^{-1}}
\end{aligned}
$$

We shall identify $\mathrm{U}_{q}\left(\mathfrak{s l}_{n}\right)$ with a subalgebra of $\mathrm{U}_{q}\left(\mathfrak{g l}_{n}\right)$ via this embedding.

We let $\mathrm{U}_{q}^{-}, \mathrm{U}_{q}^{+}$and $\mathrm{U}_{q}^{0}$ denote the subalgebras of $\mathrm{U}_{q}\left(\mathfrak{g l}_{n}\right)$ respectively generated by the $t_{i j}$ with $i>j$, the $\bar{t}_{i j}$ with $i<j$ and the $t_{i i}, \bar{t}_{i i}$ with all $i$. It is implied by [4, Proposition 9.2.2] that multiplication defines an isomorphism of vector spaces

$$
\mathrm{U}_{q}^{-} \otimes \mathrm{U}_{q}^{0} \otimes \mathrm{U}_{q}^{+} \cong \mathrm{U}_{q}\left(\mathfrak{g l}_{n}\right)
$$

The products $t_{11}^{m_{1}} \cdots t_{n n}^{m_{n}}$ with $m_{i} \in \mathbb{Z}$ form a basis of $\mathrm{U}_{q}^{0}$. Moreover, the monomials

$$
t_{n, n-1}^{k_{n, n-1}} \cdots t_{n 2}^{k_{n 2}} \cdots t_{32}^{k_{32}} t_{n 1}^{k_{n 1}} \cdots t_{21}^{k_{21}}
$$

with non-negative powers $k_{i j}$ form a basis of $\mathrm{U}_{q}^{-}$, while the monomials

$$
\bar{t}_{12}^{k_{12}} \cdots \bar{t}_{1 n}^{k_{1 n}} \bar{t}_{23}^{k_{23}} \cdots \bar{t}_{2 n}^{k_{2 n}} \cdots \bar{t}_{n-1, n}^{k_{n-1, n}}
$$

with non-negative powers $k_{i j}$ form a basis of $\mathrm{U}_{q}^{+}$.

Denote by $\mathrm{Z}_{q}$ the center of the algebra $\mathrm{U}_{q}\left(\mathfrak{g l}_{n}\right)$. Due to the isomorphism (2.10), any $z \in \mathrm{Z}_{q}$ can be regarded as an element of $\mathrm{U}_{q}^{-} \otimes \mathrm{U}_{q}^{0} \otimes \mathrm{U}_{q}^{+}$. Denote by $\chi(z)$ the projection of $z$ to the subalgebra $\mathrm{U}_{q}^{0}$ so that $\chi(z)-z$ belongs to the left ideal of $\mathrm{U}_{q}\left(\mathfrak{g l}_{n}\right)$ generated by the elements $\bar{t}_{i j}$ with $i<j$. The map

$$
\chi: \mathrm{Z}_{q} \rightarrow \mathrm{U}_{q}^{0}
$$

is an algebra homomorphism called the Harish-Chandra homomorphism. This homomorphism is injective and its image is the subalgebra of $\mathrm{U}_{q}^{0}$ generated by the symmetric polynomials in $x_{1}^{2}, \ldots, x_{n}^{2}$ and the polynomial $x_{1}^{-1} \cdots x_{n}^{-1}$, where $x_{i}=q^{-i+1} t_{i i}$. Moreover, those polynomials of total degree zero in $x_{1}, \ldots, x_{n}$ form the image of the center of the subalgebra $\mathrm{U}_{q}\left(\mathfrak{s l}_{n}\right)$ under the Harish-Chandra homomorphism; see e.g. [4, Proposition 9.2.5] and [13, Section 6.3.4]. 
Now consider the Lie algebra of Laurent polynomials $\mathfrak{g l}_{n}\left[\lambda, \lambda^{-1}\right]$ in an indeterminate $\lambda$. We denote it by $\widehat{\mathfrak{g l}}_{n}$ for brevity. The quantum affine algebra $\mathrm{U}_{q}\left(\widehat{\mathfrak{g l}}_{n}\right)$ is a deformation of the universal enveloping algebra $\mathrm{U}\left(\widehat{\mathfrak{g l}}_{n}\right)$. By definition, $\mathrm{U}_{q}\left(\widehat{\mathfrak{g l}}_{n}\right)$ has countably many generators $t_{i j}^{(r)}$ and $\bar{t}_{i j}^{(r)}$ where $1 \leqslant i, j \leqslant n$ and $r$ runs over nonnegative integers. They are combined into the matrices

$$
T(u)=\sum_{i, j=1}^{n} t_{i j}(u) \otimes E_{i j}, \quad \bar{T}(u)=\sum_{i, j=1}^{n} \bar{t}_{i j}(u) \otimes E_{i j},
$$

where $t_{i j}(u)$ and $\bar{t}_{i j}(u)$ are formal series in $u^{-1}$ and $u$, respectively:

$$
t_{i j}(u)=\sum_{r=0}^{\infty} t_{i j}^{(r)} u^{-r}, \quad \bar{t}_{i j}(u)=\sum_{r=0}^{\infty} \bar{t}_{i j}^{(r)} u^{r} .
$$

The defining relations are

$$
\begin{aligned}
& t_{i j}^{(0)}=\bar{t}_{j i}^{(0)}=0, \quad 1 \leqslant i<j \leqslant n, \\
& t_{i i}^{(0)} \bar{t}_{i i}^{(0)}=\bar{t}_{i i}^{(0)} t_{i i}^{(0)}=1, \quad 1 \leqslant i \leqslant n, \\
& R(u, v) T_{1}(u) T_{2}(v)=T_{2}(v) T_{1}(u) R(u, v), \\
& R(u, v) \bar{T}_{1}(u) \bar{T}_{2}(v)=\bar{T}_{2}(v) \bar{T}_{1}(u) R(u, v), \\
& R(u, v) \bar{T}_{1}(u) T_{2}(v)=T_{2}(v) \bar{T}_{1}(u) R(u, v),
\end{aligned}
$$

where we have used the notation of $(2.3)$ and $R(u, v)=R^{q}(u, v)$ is the trigonometric $R$-matrix given by

$$
\begin{aligned}
R^{q}(u, v)= & (u-v) \sum_{i \neq j} E_{i i} \otimes E_{j j}+\left(q^{-1} u-q v\right) \sum_{i} E_{i i} \otimes E_{i i} \\
& +\left(q^{-1}-q\right) u \sum_{i>j} E_{i j} \otimes E_{j i}+\left(q^{-1}-q\right) v \sum_{i<j} E_{i j} \otimes E_{j i} .
\end{aligned}
$$

It satisfies the Yang-Baxter equation

$$
R_{12}(u, v) R_{13}(u, w) R_{23}(v, w)=R_{23}(v, w) R_{13}(u, w) R_{12}(u, v),
$$

where both sides take values in End $\mathbb{C}^{n} \otimes$ End $\mathbb{C}^{n} \otimes$ End $\mathbb{C}^{n}$ and the indices indicate the copies of End $\mathbb{C}^{n}$, e.g., $R_{12}(u, v)=R(u, v) \otimes 1$ etc.; see e.g. [6, 8] for more details on the structure of $\mathrm{U}_{q}\left(\widehat{\mathfrak{g l}}_{n}\right)$. Note that it is more common in the literature to use the notation $\mathrm{U}_{q}\left(\widehat{\mathfrak{g l}}_{n}\right)$ for the centrally extended quantum affine algebra. In this paper, however, we are mainly concerned with finite-dimensional representations of the quantum affine algebra where the central element acts trivially, so that the notation should not cause a confusion.

The quantized enveloping algebra $\mathrm{U}_{q}\left(\mathfrak{g l}_{n}\right)$ is a natural subalgebra of $\mathrm{U}_{q}\left(\widehat{\mathfrak{g l}}_{n}\right)$ defined by the embedding

$$
t_{i j} \mapsto t_{i j}^{(0)}, \quad \bar{t}_{i j} \mapsto \bar{t}_{i j}^{(0)} .
$$

Moreover, there is an algebra homomorphism $\mathrm{U}_{q}\left(\widehat{\mathfrak{g l}}_{n}\right) \rightarrow \mathrm{U}_{q}\left(\mathfrak{g l}_{n}\right)$ called the evaluation homomorphism defined by

$$
T(u) \mapsto T-\bar{T} u^{-1}, \quad \bar{T}(u) \mapsto \bar{T}-T u .
$$

Now we recall the well-known construction of the quantum determinants for the algebra $\mathrm{U}_{q}\left(\widehat{\mathfrak{g l}}_{n}\right)$; see e.g. [5, 11, 30]. Consider the multiple tensor product $\mathrm{U}_{q}\left(\widehat{\mathfrak{g l}}_{n}\right) \otimes\left(\text { End } \mathbb{C}^{n}\right)^{\otimes r}$ and use the notation of (2.14). Then we have the following corollary of (2.16) and (2.14):

$$
R\left(u_{1}, \ldots, u_{r}\right) T_{1}\left(u_{1}\right) \cdots T_{r}\left(u_{r}\right)=T_{r}\left(u_{r}\right) \cdots T_{1}\left(u_{1}\right) R\left(u_{1}, \ldots, u_{r}\right),
$$


where

$$
R\left(u_{1}, \ldots, u_{r}\right)=\prod_{i<j} R_{i j}\left(u_{i}, u_{j}\right)
$$

with the product taken in the lexicographical order on the pairs $(i, j)$. The proof of $(2.19)$ is exactly the same as for the Yangians; see e.g. [20]. Furthermore, consider the $q$-permutation operator $P^{q} \in$ End $\left(\mathbb{C}^{n} \otimes \mathbb{C}^{n}\right)$ defined by

$$
P^{q}=\sum_{i} E_{i i} \otimes E_{i i}+q \sum_{i>j} E_{i j} \otimes E_{j i}+q^{-1} \sum_{i<j} E_{i j} \otimes E_{j i} .
$$

For $q=1$ the operator $P^{q}$ turns into the usual permutation operator

$$
P=\sum_{i, j} E_{i j} \otimes E_{j i}
$$

The action of symmetric group $\mathfrak{S}_{r}$ on the space $\left(\mathbb{C}^{n}\right)^{\otimes r}$ can be defined by setting $s_{i} \mapsto P_{s_{i}}^{q}:=$ $P_{i, i+1}^{q}$ for $i=1, \ldots, r-1$, where $s_{i}$ denotes the transposition $(i, i+1)$. If $\sigma=s_{i_{1}} \cdots s_{i_{l}}$ is a reduced decomposition of an element $\sigma \in \mathfrak{S}_{r}$ we set $P_{\sigma}^{q}=P_{s_{i_{1}}}^{q} \cdots P_{s_{i_{l}}}^{q}$. We note some useful formulas for the action of $P_{\sigma}^{q}$ on $\left(\mathbb{C}^{n}\right)^{\otimes r}$. Denote by $e_{1}, \ldots, e_{n}$ the canonical basis vectors of $\mathbb{C}^{n}$. Then for any indices $a_{1}<\cdots<a_{r}$ we have

$$
P_{\sigma}^{q}\left(e_{a_{1}} \otimes \cdots \otimes e_{a_{r}}\right)=q^{l(\sigma)} e_{a_{\sigma^{-1}(1)}} \otimes \cdots \otimes e_{a_{\sigma^{-1}(r)}},
$$

where $l(\sigma)$ denotes the length of the permutation $\sigma$. This implies a more general formula: for any $\tau \in \mathfrak{S}_{r}$ we have

$$
P_{\sigma}^{q}\left(e_{a_{\tau(1)}} \otimes \cdots \otimes e_{a_{\tau(r)}}\right)=q^{l\left(\sigma \tau^{-1}\right)-l(\tau)} e_{a_{\tau \sigma^{-1}(1)}} \otimes \cdots \otimes e_{a_{\tau \sigma^{-1}(r)}} .
$$

We denote by $A_{r}^{q}$ the $q$-antisymmetrizer

$$
A_{r}^{q}=\sum_{\sigma \in \mathfrak{S}_{r}} \operatorname{sgn} \sigma \cdot P_{\sigma}^{q}
$$

The above formulas imply that form any $\tau \in \mathfrak{S}_{r}$ we have

$$
A_{r}^{q}\left(e_{a_{\tau(1)}} \otimes \cdots \otimes e_{a_{\tau(r)}}\right)=(-q)^{-l(\tau)} A_{r}^{q}\left(e_{a_{1}} \otimes \cdots \otimes e_{a_{r}}\right) .
$$

We have the relation in End $\left(\mathbb{C}^{n}\right)^{\otimes r}$ :

$$
R\left(1, q^{-2}, \ldots, q^{-2 r+2}\right)=\prod_{0 \leqslant i<j \leqslant r-1}\left(q^{-2 i}-q^{-2 j}\right) A_{r}^{q} .
$$

Now (2.19) implies

$$
A_{r}^{q} T_{1}(u) \cdots T_{r}\left(q^{-2 r+2} u\right)=T_{r}\left(q^{-2 r+2} u\right) \cdots T_{1}(u) A_{r}^{q}
$$

which equals

$$
\sum_{a_{i}, b_{i}} t_{b_{1} \cdots b_{r}}^{a_{1} \cdots a_{r}}(u) \otimes E_{a_{1} b_{1}} \otimes \cdots \otimes E_{a_{r} b_{r}}
$$

for some elements $t_{b_{1} \cdots b_{r}}^{a_{1} \cdots a_{r}}(u)$ of $\mathrm{U}_{q}\left(\widehat{\mathfrak{g l}}_{n}\right)\left[\left[u^{-1}\right]\right]$ which we call the quantum minors. They can be given by the following formulas which are immediate from the definition. If $a_{1}<\cdots<a_{r}$ then

$$
t_{b_{1} \cdots b_{r}}^{a_{1} \cdots a_{r}}(u)=\sum_{\sigma \in \mathfrak{S}_{r}}(-q)^{-l(\sigma)} \cdot t_{a_{\sigma(1)} b_{1}}(u) \cdots t_{a_{\sigma(r)} b_{r}}\left(q^{-2 r+2} u\right)
$$


and for any $\tau \in \mathfrak{S}_{r}$ we have

$$
t_{b_{1} \cdots b_{r}}^{a_{\tau(1)} \cdots a_{\tau(r)}}(u)=(-q)^{l(\tau)} t_{b_{1} \cdots b_{r}}^{a_{1} \cdots a_{r}}(u)
$$

If $b_{1}<\cdots<b_{r}$ (and the $a_{i}$ are arbitrary) then

$$
t_{b_{1} \cdots b_{r}}^{a_{1} \cdots a_{r}}(u)=\sum_{\sigma \in \mathfrak{S}_{r}}(-q)^{l(\sigma)} \cdot t_{a_{r} b_{\sigma(r)}}\left(q^{-2 r+2} u\right) \cdots t_{a_{1} b_{\sigma(1)}}(u)
$$

and for any $\tau \in \mathfrak{S}_{r}$ we have

$$
t_{b_{\tau(1)} \cdots b_{\tau(r)}}^{a_{1} \cdots a_{r}}(u)=(-q)^{-l(\tau)} t_{b_{1} \cdots b_{r}}^{a_{1} \cdots a_{r}}(u) .
$$

Moreover, the quantum minor is zero if two top or two bottom indices are equal.

As the defining relations for the generators $\bar{t}_{i j}^{(r)}$ have the same matrix form as for the $t_{i j}^{(r)}$, the above argument can be applied to define the respective quantum minors $\bar{t}_{b_{1} \cdots b_{r}}^{a_{1} \cdots a_{r}}(u) \in$ $\mathrm{U}_{q}\left(\widehat{\mathfrak{g l}}_{n}\right)[[u]]$. They are given by the same formulas (2.26) and (2.28), where the $t_{i j}(u)$ are respectively replaced with $\bar{t}_{i j}(u)$. Indeed, we have the relation

$$
A_{r}^{q} \bar{T}_{1}(u) \cdots \bar{T}_{r}\left(q^{-2 r+2} u\right)=\bar{T}_{r}\left(q^{-2 r+2} u\right) \cdots \bar{T}_{1}(u) A_{r}^{q}
$$

analogous to (2.24) so that both sides are equal to

$$
\sum_{a_{i}, b_{i}} \bar{t}_{b_{1} \cdots b_{r}}^{a_{1} \cdots a_{r}}(u) \otimes E_{a_{1} b_{1}} \otimes \cdots \otimes E_{a_{r} b_{r}}
$$

Furthermore, for any indices $i, j$ we have the well known relations which are deduced from (2.19):

$$
\left[t_{c_{i} d_{j}}(u), t_{d_{1} \cdots d_{r}}^{c_{1} \cdots c_{r}}(v)\right]=0, \quad\left[t_{c_{i} d_{j}}(u), \bar{t}_{d_{1} \cdots d_{r}}^{c_{1} \cdots c_{r}}(v)\right]=0
$$

and the same holds with $t_{c_{i} d_{j}}(u)$ replaced by $\bar{t}_{c_{i} d_{j}}(u)$.

The quantum determinants of the matrices $T(u)$ and $\bar{T}(u)$ are respectively defined by the relations

$$
\operatorname{qdet} T(u)=t_{1 \cdots n}^{1 \cdots n}(u), \quad q \operatorname{det} \bar{T}(u)=\bar{t}_{1 \cdots n}^{1 \cdots n}(u)
$$

Write

$$
\operatorname{qdet} T(u)=\sum_{k=0}^{\infty} d_{k} u^{-k}, \quad q \operatorname{qdet} \bar{T}(u)=\sum_{k=0}^{\infty} \bar{d}_{k} u^{k}, \quad d_{k}, \bar{d}_{k} \in \mathrm{U}_{q}\left(\widehat{\mathfrak{g l}}_{n}\right) .
$$

Due to the property $(2.32)$, the coefficients $d_{k}$ and $\bar{d}_{k}$ belong to the center of the algebra $\mathrm{U}_{q}\left(\widehat{\mathfrak{g l}}_{n}\right)$. Furthermore, we have the relation $d_{0} \bar{d}_{0}=1$ which is implied by $(2.14)$.

Note that for any nonzero constant $\alpha$, the mapping

$$
T(u) \mapsto T(\alpha u), \quad \bar{T}(u) \mapsto \bar{T}(\alpha u)
$$

defines an automorphism of the algebra $\mathrm{U}_{q}\left(\widehat{\mathfrak{g l}}_{n}\right)$. 


\section{Quantum Sylvester theorem}

Suppose that $A=\left[a_{i j}\right]$ is a numerical $n \times n$ matrix and let $1 \leqslant m \leqslant n$. For any indices $i, j=1, \ldots, m$ introduce the minors $c_{i j}$ of $A$ corresponding to the rows $i, m+1, \ldots, n$ and columns $j, m+1, \ldots, n$ so that

$$
c_{i j}=a_{j, m+1, \ldots, n}^{i, m+1, \ldots, n} .
$$

Let $A_{\mathcal{Q Q}}$ be the submatrix of $A$ whose rows and columns are numbered by the elements of the set $\mathcal{Q}=\{m+1, \ldots, n\}$. The classical Sylvester theorem provides a formula for the determinant of the matrix $C=\left[c_{i j}\right]$ :

$$
\operatorname{det} C=\operatorname{det} A \cdot\left(\operatorname{det} A_{\mathcal{Q Q}}\right)^{m-1} .
$$

We shall give a $q$-analogue of this theorem where minors of $A$ are replaced by quantum minors of the matrices $T(u)$ or $\bar{T}(u)$. Following [15], for its proof we employ a certain $q$-analogue of the complimentary minor identity.

We shall also be using the algebra $\mathrm{U}_{q^{-1}}\left(\widehat{\mathfrak{g l}}_{n}\right)$. In order to distinguish its generators from those of the algebra $\mathrm{U}_{q}\left(\widehat{\mathfrak{g l}}_{n}\right)$ we mark them by the symbol ${ }^{\circ}$. In particular, $t_{i j}^{\circ}(u)$ and $\bar{t}_{i j}^{\circ}(u)$ will denote the corresponding generating series while $T^{\circ}(u)$ and $\bar{T}^{\circ}(u)$ will stand for the generator matrices.

Proposition 3.1. The mapping

$$
\omega_{n}: T(u) \mapsto T^{\circ}(u)^{-1}, \quad \bar{T}(u) \mapsto \bar{T}^{\circ}(u)^{-1}
$$

defines an isomorphism $\omega_{n}: \mathrm{U}_{q}\left(\widehat{\mathfrak{g l}}_{n}\right) \rightarrow \mathrm{U}_{q^{-1}}\left(\widehat{\mathfrak{g l}}_{n}\right)$.

Proof. The matrix $T^{\circ}(u)$ satisfies the relation

$$
R^{q^{-1}}(u, v) T_{1}^{\circ}(u) T_{2}^{\circ}(v)=T_{2}^{\circ}(v) T_{1}^{\circ}(u) R^{q^{-1}}(u, v) .
$$

Multiplying both sides on the left by the product $T_{1}^{\circ}(u)^{-1} T_{2}^{\circ}(v)^{-1}$ and on the right by the product $T_{2}^{\circ}(v)^{-1} T_{1}^{\circ}(u)^{-1}$, then conjugating by $P$ and swapping the parameters $u$ and $v$ gives

$$
P R^{q^{-1}}(v, u) P T_{1}^{\circ}(u)^{-1} T_{2}^{\circ}(v)^{-1}=T_{2}^{\circ}(v)^{-1} T_{1}^{\circ}(u)^{-1} P R^{q^{-1}}(v, u) P .
$$

Observing that $P R^{q^{-1}}(v, u) P=-R^{q}(u, v)$, and applying the same argument to the two remaining matrix relations in (2.14), we conclude that $\omega_{n}$ defines a homomorphism. This map is obviously invertible with the inverse given by

$$
\omega_{n}^{-1}: T^{\circ}(u) \mapsto T(u)^{-1}, \quad \bar{T}^{\circ}(u) \mapsto \bar{T}(u)^{-1}
$$

so that $\omega_{n}$ is an isomorphism.

Suppose now that the integer $m$ is such that $0 \leqslant m \leqslant n$. For any two subsets

$$
\mathcal{P}=\left\{i_{1}, \ldots, i_{m}\right\} \quad \text { and } \quad \mathcal{Q}=\left\{j_{1}, \ldots, j_{m}\right\}
$$

of $\{1, \ldots, n\}$ with cardinality $m$ let

$$
\overline{\mathcal{P}}=\left\{i_{m+1}, \ldots, i_{n}\right\} \quad \text { and } \quad \overline{\mathcal{Q}}=\left\{j_{m+1}, \ldots, j_{n}\right\}
$$

be their set complements in $\{1, \ldots, n\}$. We assume that

$$
i_{1}<\cdots<i_{m} \quad \text { and } \quad j_{1}<\cdots<j_{m}, \quad i_{m+1}<\cdots<i_{n} \quad \text { and } \quad j_{m+1}<\cdots<j_{n} \text {. }
$$


For any $n \times n$ matrix $X$, we will denote by $X_{\mathcal{P} \mathcal{Q}}$ the submatrix whose rows and columns are numbered by the elements of the sets $\mathcal{P}$ and $\mathcal{Q}$ respectively.

Each of the sequences $i_{1}, \ldots, i_{n}$ and $j_{1}, \ldots, j_{n}$ above is a permutation of the sequence $1, \ldots, n$. Denote these two permutations by $\mathbf{i}$ and $\mathbf{j}$ respectively. If $A$ is a $n \times n$ matrix with complex entries and $B$ is the inverse matrix, then the following identity for complementary minors of $A$ and $B$ holds:

$$
\operatorname{det} A \cdot b_{i_{m+1} \ldots i_{n}}^{j_{m+1} \ldots j_{n}}=\operatorname{sgn} \mathbf{i} \cdot \operatorname{sgn} \mathbf{j} \cdot a_{j_{1} \ldots j_{m}}^{i_{1} \ldots i_{m}}
$$

Here $a_{j_{1} \ldots j_{m}}^{i_{1} \ldots i_{m}}$ is the minor of $A$ corresponding to the submatrix $A_{\mathcal{P} \mathcal{Q}}$, and $b_{i_{m+1} \ldots i_{n}}^{j_{m+1} j_{n}}$ is the minor of $B$ corresponding to the submatrix $B \overline{\mathcal{Q}} \overline{\mathcal{P}}$. We now give analogues of the identity (3.1) for the matrices $T(u)$ and $\bar{T}(u)$.

Theorem 3.2. We have the identities

$$
\begin{aligned}
& \mathrm{qdet} T(u) \cdot \omega_{n}^{-1}\left(t^{\circ{ }_{i_{m+1} \ldots i_{n}}^{j_{m+1} \ldots j_{n}}}\left(q^{-2 n+2} u\right)\right)=(-q)^{l(\mathbf{j})-l(\mathbf{i})} \cdot t_{j_{1} \ldots j_{m}}^{i_{1} \ldots i_{m}}(u), \\
& q \operatorname{det} \bar{T}(u) \cdot \omega_{n}^{-1}\left(\bar{t}^{\circ j_{m+1} \ldots j_{n}}\left(q_{i_{m+1} \ldots i_{n}} q^{-2 n+2} u\right)\right)=(-q)^{l(\mathbf{j})-l(\mathbf{i})} \cdot \bar{t}_{j_{1} \ldots j_{m}}^{i_{1} \ldots i_{m}}(u) \text {, }
\end{aligned}
$$

where $t^{\circ{ }_{i_{m+1} \ldots i_{n}}}(v)$ and $\bar{t}^{\circ j_{m+1} \ldots j_{n}}{ }_{i_{m+1} \ldots i_{n}}^{j_{m+1}}(v)$ denote the quantum minors in the quantum affine algebra $\mathrm{U}_{q^{-1}}\left(\widehat{\mathfrak{g l}}_{n}\right)$.

Proof. Both identities are verified in the same way so we only consider the first one. By the definition of the quantum determinant,

$$
\operatorname{qdet} T(u) A_{n}^{q}=A_{n}^{q} T_{1} \cdots T_{n},
$$

where $T_{i}=T_{i}\left(q^{-2 i+2} u\right)$ for $i=1, \ldots, n$. Let us multiply both sides of $(3.2)$ by $T_{n}^{-1} \cdots T_{m+1}^{-1}$ from the right. Then (3.2) takes the form

$$
\operatorname{qdet} T(u) A_{n}^{q} T_{n}^{-1} \cdots T_{m+1}^{-1}=A_{n}^{q} T_{1} \cdots T_{m} .
$$

Now we apply both sides of (3.3) to the basis vector

$$
e_{j_{1}} \otimes \cdots \otimes e_{j_{m}} \otimes e_{i_{m+1}} \otimes \cdots \otimes e_{i_{n}} \in\left(\mathbb{C}^{n}\right)^{\otimes n} .
$$

The right hand side gives

$$
A_{n}^{q} \sum_{a_{1}, \ldots, a_{m}} t_{a_{1} j_{1}}(u) \cdots t_{a_{m} j_{m}}\left(q^{-2 m+2} u\right) e_{a_{1}} \otimes \cdots \otimes e_{a_{m}} \otimes e_{i_{m+1}} \otimes \cdots \otimes e_{i_{n}} .
$$

The summation here can obviously be restricted to the sequences $a_{1}, \ldots, a_{m}$ which are permutations of the sequence $i_{1}, \ldots, i_{m}$. Consider the vector $A_{n}^{q}\left(e_{1} \otimes \cdots \otimes e_{n}\right)$. The sum (3.5) is proportional to this vector, with the coefficient

$$
(-q)^{-l(\mathbf{i})} \cdot t_{j_{1} \ldots j_{m}}^{i_{1} \ldots i_{m}}(u)
$$

We shall use the notation $t_{i j}^{\prime}(u)$ for the entries of the matrix $T^{-1}(u)$. By applying the left hand side of (3.3) to the basis vector (3.4), we obtain

$$
A_{n}^{q} \sum_{b_{m+1}, \ldots, b_{n}} t_{b_{n} i_{n}}^{\prime}\left(q^{-2 n+2} u\right) \cdots t_{b_{m+1} i_{m+1}}^{\prime}\left(q^{-2 m} u\right) e_{j_{1}} \otimes \cdots \otimes e_{j_{m}} \otimes e_{b_{m+1}} \otimes \cdots \otimes e_{b_{n}}
$$


Here the coefficient of $A_{n}^{q}\left(e_{1} \otimes \cdots \otimes e_{n}\right)$ equals

$$
(-q)^{-l(\mathbf{j})} \cdot \sum_{\sigma}(-q)^{-l(\sigma)} t_{j_{\sigma(n)} i_{n}}^{\prime}\left(q^{-2 n+2} u\right) \cdots t_{j_{\sigma(m+1)} i_{m+1}}^{\prime}\left(q^{-2 m} u\right)
$$

summed over permutations $\sigma$ of the set $\{m+1, \ldots, n\}$. This coefficient can be written as

$$
(-q)^{-l(\mathbf{j})} \cdot \omega_{n}^{-1}\left(t^{\circ} \underset{i_{m+1} \ldots i_{n}}{j_{m+1} \ldots j_{n}}\left(q^{-2 n+2} u\right)\right)
$$

Thus the desired identity follows from (3.3).

Corollary 3.3. We have the identities

$$
\begin{aligned}
& q \operatorname{det} T(u) \cdot \omega_{n}^{-1}\left(q \operatorname{det} T^{\circ}\left(q^{-2 n+2} u\right)\right)=1, \\
& q \operatorname{det} \bar{T}(u) \cdot \omega_{n}^{-1}\left(q \operatorname{det} \bar{T}^{\circ}\left(q^{-2 n+2} u\right)\right)=1,
\end{aligned}
$$

where $q \operatorname{det} T^{\circ}(v)$ and $\operatorname{qdet} \bar{T}^{\circ}(v)$ denote the quantum determinants in $\mathrm{U}_{q^{-1}}\left(\widehat{\mathfrak{g l}}_{n}\right)$.

For any $0 \leqslant m \leqslant n$ introduce the homomorphism $\imath_{m, n}$

$$
\imath_{m, n}: \mathrm{U}_{q^{-1}}\left(\widehat{\mathfrak{g l}}_{m}\right) \rightarrow \mathrm{U}_{q^{-1}}\left(\widehat{\mathfrak{g l}}_{n}\right)
$$

which takes the coefficients of the series $t_{i j}^{\circ}(u)$ and $\bar{t}_{i j}^{\circ}(u)$ to the respective elements of $\mathrm{U}_{q^{-1}}\left(\widehat{\mathfrak{g l}}_{n}\right)$ with the same name. Consider the composition

$$
\psi_{m, n}=\omega_{n}^{-1} \circ \imath_{m, n} \circ \omega_{m}
$$

which is an algebra homomorphism

$$
\psi_{m, n}: \mathrm{U}_{q}\left(\widehat{\mathfrak{g l}}_{m}\right) \rightarrow \mathrm{U}_{q}\left(\widehat{\mathfrak{g l}}_{n}\right)
$$

The action of $\psi_{m, n}$ on the quantum minors is described in the next lemma.

Lemma 3.4. We have

$$
\psi_{m, n}: t_{b_{1} \ldots b_{r}}^{a_{1} \ldots a_{r}}(u) \mapsto\left(t_{m+1 \ldots n}^{m+1 \ldots n}\left(q^{2 n-2 m} u\right)\right)^{-1} \cdot t_{b_{1} \ldots b_{r}, m+1 \ldots n}^{a_{1} \ldots a_{r}, m+1 \ldots n}\left(q^{2 n-2 m} u\right),
$$

where $a_{i}, b_{i} \in\{1, \ldots, m\}$, and the same formula holds with the minors in the $t_{i j}(u)$ replaced with the respective minors in the $\bar{t}_{i j}(u)$.

Proof. Due to (2.27) and (2.29) we may assume that $a_{1}<\cdots<a_{r}$ and $b_{1}<\cdots<b_{r}$. By Theorem 3.2 and Corollary 3.3 we have

$$
\omega_{m}: t_{b_{1} \ldots b_{r}}^{a_{1} \ldots a_{r}}(u) \mapsto(-q)^{l(\mathbf{a})-l(\mathbf{b})} \cdot t^{\circ 1 \ldots m}\left(q^{-2 m+2} u\right)^{-1} \cdot t^{\circ}{ }_{a_{r+1} \ldots a_{m}}^{b_{r+1} \ldots b_{m}}\left(q^{-2 m+2} u\right),
$$

where $a_{r+1}<\cdots<a_{m}$ and $b_{r+1}<\cdots<b_{m}$ are respective complementary elements to the sets $\left\{a_{1}, \ldots, a_{r}\right\}$ and $\left\{b_{1}, \ldots, b_{r}\right\}$ in $\{1, \ldots, m\}$, while $\mathbf{a}$ and $\mathbf{b}$ denote the permutations $\left(a_{1}, \ldots, a_{m}\right)$ and $\left(b_{1}, \ldots, b_{m}\right)$ of the sequence $(1, \ldots, m)$. Now, applying the homomorphism $\imath_{m, n}$ we may regard the quantum minors on the right hand side as series with coefficients in the algebra $\mathrm{U}_{q^{-1}}\left(\widehat{\mathfrak{g l}}_{n}\right)$. The proof is completed by another application of Theorem 3.2. The same argument proves the corresponding formula for the minors in the $\bar{t}_{i j}(u)$.

In particular, we have the following explicit formulas for the images of the generators of $\mathrm{U}_{q}\left(\widehat{\mathfrak{g l}}_{n}\right)$. 
Corollary 3.5. For any $1 \leqslant i, j \leqslant m$, we have

$$
\psi_{m, n}: t_{i j}(u) \mapsto\left(t_{m+1 \ldots n}^{m+1 \ldots n}\left(q^{2 n-2 m} u\right)\right)^{-1} \cdot t_{j, m+1 \ldots n}^{i, m+1 \ldots n}\left(q^{2 n-2 m} u\right),
$$

and the same formula holds with the image of the $\bar{t}_{i j}(u)$ where the minors in the $t_{i j}(u)$ are replaced with the respective minors in the $\bar{t}_{i j}(u)$.

The subalgebra of $\mathrm{U}_{q}\left(\widehat{\mathfrak{g l}}_{n}\right)$ generated by the elements $t_{i j}^{(r)}$ and $\bar{t}_{i i}^{(0)}$ was studied e.g. in $[5,25,30]$. We call it the $q$-Yangian and denote by $\mathrm{Y}_{q}\left(\mathfrak{g l}_{n}\right)$. For any $1 \leqslant i, j \leqslant m$, introduce the following series with coefficients in $\mathrm{Y}_{q}\left(\mathfrak{g l}_{n}\right)$

$$
t_{i j}^{b}(u)=t_{j, m+1 \ldots n}^{i, m+1 \ldots n}(u)
$$

and combine them into the matrix $T^{b}(u)=\left[t_{i j}^{b}(u)\right]$. Let $T(u)_{\mathcal{Q Q}}$ be the submatrix of $T(u)$ whose rows and columns are numbered by the elements of the set $\mathcal{Q}=\{m+1, \ldots, n\}$.

The following is an analogue of the Sylvester theorem for the $q$-Yangian.

Theorem 3.6. The mapping

$$
t_{i j}(u) \mapsto t_{i j}^{b}(u), \quad 1 \leqslant i, j \leqslant m,
$$

defines a homomorphism $\mathrm{Y}_{q}\left(\mathfrak{g l}_{m}\right) \rightarrow \mathrm{Y}_{q}\left(\mathfrak{g l}_{n}\right)$. Moreover, we have the identity

$$
\operatorname{qdet} T^{b}(u)=\operatorname{qdet} T(u) \cdot \operatorname{qdet} T\left(q^{-2} u\right)_{\mathcal{Q Q}} \cdots \operatorname{qdet} T\left(q^{-2 m+2} u\right)_{\mathcal{Q Q}} \text {. }
$$

Proof. By Corollary 3.5, we have

$$
\psi_{m, n}: t_{i j}\left(q^{-2 n+2 m} u\right) \mapsto\left(\operatorname{qdet} T(u)_{\mathcal{Q Q}}\right)^{-1} \cdot t_{i j}^{b}(u) .
$$

Relation (2.32) implies that the coefficients of the series qdet $T(u)_{\mathcal{Q Q}}$ commute with those of the series $t_{i j}^{b}(v)$. Hence, since $\psi_{m, n}$ is a homomorphism, we can conclude by the application of the automorphism (2.35) that the mapping (3.8) defines a homomorphism. Furthermore, by Lemma 3.4,

$$
\psi_{m, n}: t_{1 \ldots m}^{1 \ldots m}\left(q^{-2 n+2 m} u\right) \mapsto\left(\operatorname{qdet} T(u)_{\mathcal{Q Q}}\right)^{-1} \cdot \operatorname{qdet} T(u) .
$$

On the other hand, expanding the quantum minor, we obtain from (3.9) that

$$
\psi_{m, n}: t_{1 \ldots m}^{1 \ldots m}\left(q^{-2 n+2 m} u\right) \mapsto\left(\operatorname{qdet} T(u)_{\mathcal{Q Q}} \cdots \operatorname{qdet} T\left(q^{-2 m+2} u\right)_{\mathcal{Q Q}}\right)^{-1} \cdot \operatorname{qdet} T^{b}(u),
$$

completing the proof of the desired identity for $q \operatorname{det} T^{b}(u)$.

We shall also need a dual version of the quantum Sylvester theorem. Let $m \geqslant 0$ and $n \geqslant 1$. Instead of the homomorphism $\imath_{m, n}$ defined in (3.6), consider another homomorphism

$$
\jmath_{m}: \mathrm{U}_{q^{-1}}\left(\widehat{\mathfrak{g l}}_{n}\right) \rightarrow \mathrm{U}_{q^{-1}}\left(\widehat{\mathfrak{g l}}_{m+n}\right)
$$

which takes the coefficients of the series $t_{i j}^{\circ}(u)$ and $\bar{t}_{i j}^{\circ}(u)$ to the respective coefficients of the series $t_{m+i, m+j}^{\circ}(u)$ and $\bar{t}_{m+i, m+j}^{\circ}(u)$. Consider the composition

$$
\phi_{m}=\omega_{m+n}^{-1} \circ \jmath_{m} \circ \omega_{n} .
$$

Then $\phi_{m}$ is an algebra homomorphism

$$
\phi_{m}: \mathrm{U}_{q}\left(\widehat{\mathfrak{g l}}_{n}\right) \rightarrow \mathrm{U}_{q}\left(\widehat{\mathfrak{g l}}_{m+n}\right) .
$$

The corresponding version of Lemma 3.4 now takes the form 
Lemma 3.7. We have

$$
\phi_{m}: t_{b_{1} \ldots b_{r}}^{a_{1} \ldots a_{r}}(u) \mapsto\left(t_{1 \ldots m}^{1 \ldots m}\left(q^{2 m} u\right)\right)^{-1} \cdot t_{1 \ldots m, m+b_{1} \ldots m+b_{r}}^{1 \ldots m, m+a_{1} \ldots m+a_{r}}\left(q^{2 m} u\right),
$$

where $a_{i}, b_{i} \in\{1, \ldots, n\}$, and the same formula holds with the minors in the $t_{i j}(u)$ replaced with the respective minors in the $\bar{t}_{i j}(u)$.

For any indices $1 \leqslant i, j \leqslant n$ introduce the following series with coefficients in the $q$-Yangian $\mathrm{Y}_{q}\left(\mathfrak{g l}_{m+n}\right)$,

$$
t_{i j}^{\sharp}(u)=t_{1 \ldots m, m+j}^{1 \ldots m, m+i}(u),
$$

and combine them into the matrix $T^{\sharp}(u)=\left[t_{i j}^{\sharp}(u)\right]$. Let $T(u)_{\mathcal{P P}}$ be the submatrix of $T(u)$ whose rows and columns are numbered by the elements of the set $\mathcal{P}=\{1, \ldots, m\}$. The following theorem is proved in the same way as Theorem 3.6.

Theorem 3.8. The mapping

$$
t_{i j}(u) \mapsto t_{i j}^{\sharp}(u), \quad 1 \leqslant i, j \leqslant n,
$$

defines a homomorphism $\mathrm{Y}_{q}\left(\mathfrak{g l}_{n}\right) \rightarrow \mathrm{Y}_{q}\left(\mathfrak{g l}_{m+n}\right)$. Moreover, we have the identity

$$
\operatorname{qdet} T^{\sharp}(u)=q \operatorname{det} T(u) \cdot q \operatorname{det} T\left(q^{-2} u\right)_{\mathcal{P P}} \cdots \operatorname{qdet} T\left(q^{-2 n+2} u\right)_{\mathcal{P P}} \text {. }
$$

Remark 1. The obvious analogues of Theorems 3.6 and 3.8 for the subalgebras of $\mathrm{U}_{q}\left(\widehat{\mathfrak{g l}}_{n}\right)$ generated by the elements $\bar{t}_{i j}^{(r)}$ and $t_{i i}$ can be proved by the same argument.

We also point out a corollary to be used in Section 4. It follows from Lemma 3.7 with the use of the automorphism (2.35).

Corollary 3.9. The mapping

$$
\begin{aligned}
& t_{i j}(u) \mapsto\left(t_{1 \ldots m}^{1 \ldots m}(u)\right)^{-1} \cdot t_{1 \ldots m, m m+j}^{1 \ldots m,}(u), \\
& \bar{t}_{i j}(u) \mapsto\left(\bar{t}_{1 \ldots m}^{1 \ldots m}(u)\right)^{-1} \cdot \bar{t}_{1 \ldots m, m, i}^{1 \ldots m+j}(u),
\end{aligned}
$$

defines a homomorphism $\mathrm{U}_{q}\left(\widehat{\mathfrak{g l}}_{n}\right) \rightarrow \mathrm{U}_{q}\left(\widehat{\mathfrak{g l}}_{m+n}\right)$. Moreover, the images of the quantum minors under the homomorphism (3.13) are found by

$$
t_{b_{1} \ldots b_{r}}^{a_{1} \ldots a_{r}}(u) \mapsto\left(t_{1 \ldots m}^{1 \ldots m}(u)\right)^{-1} \cdot t_{1 \ldots m, m+b_{1} \ldots m+b_{r}}^{1 \ldots m, m+a_{1} \ldots m+a_{r}}(u),
$$

where $a_{i}, b_{i} \in\{1, \ldots, n\}$, and the same formula holds with the minors in the $t_{i j}(u)$ replaced with the respective minors in the $\bar{t}_{i j}(u)$.

\section{Skew representations of $\mathrm{U}_{q}\left(\widehat{\mathfrak{g l}}_{n}\right)$}

Consider the quantum affine algebra $\mathrm{U}_{q}\left(\widehat{\mathfrak{g l}}_{n}\right)$ and suppose, as before, that the complex parameter $q$ is nonzero and not a root of unity. The irreducible (pseudo) highest weight representation $L(\nu(u), \bar{\nu}(u))$ of $\mathrm{U}_{q}\left(\widehat{\mathfrak{g l}}_{n}\right)$ with the (pseudo) highest weight $(\nu(u), \bar{\nu}(u))$ is generated by a nonzero vector $\zeta$ such that

$$
\begin{array}{lll}
t_{i j}(u) \zeta=0, & \bar{t}_{i j}(u) \zeta=0 & \text { for } \quad 1 \leqslant i<j \leqslant n, \\
t_{i i}(u) \zeta=\nu_{i}(u) \zeta, & \bar{t}_{i i}(u) \zeta=\bar{\nu}_{i}(u) \zeta & \text { for } \quad 1 \leqslant i \leqslant n,
\end{array}
$$


where $\nu(u)=\left(\nu_{1}(u), \ldots, \nu_{n}(u)\right)$ and $\bar{\nu}(u)=\left(\bar{\nu}_{1}(u), \ldots, \bar{\nu}_{n}(u)\right)$ are certain $n$-tuples of formal power series in $u^{-1}$ and $u$, respectively.

Suppose that there exist polynomials $P_{1}(u), \ldots, P_{n-1}(u)$ in $u$, all with constant term 1 , such that

$$
\frac{\nu_{k}(u)}{\nu_{k+1}(u)}=q^{-\operatorname{deg} P_{k}} \cdot \frac{P_{k}\left(u q^{2}\right)}{P_{k}(u)}=\frac{\bar{\nu}_{k}(u)}{\bar{\nu}_{k+1}(u)}
$$

for any $k=1, \ldots, n-1$. The first equality in (4.1) is understood in the sense that the ratio of polynomials has to be expanded as a power series in $u^{-1}$, while for the second equality the same ratio has to be expanded as a power series in $u$. Then the representation $L(\nu(u), \bar{\nu}(u))$ is finitedimensional. The $P_{k}(u)$ are called the Drinfeld polynomials of this representation. The families of Drinfeld polynomials parameterize the type 1 finite-dimensional irreducible representations of the subalgebra $\mathrm{U}_{q}\left(\widehat{\mathfrak{s l}}_{n}\right)$ of $\mathrm{U}_{q}\left(\widehat{\mathfrak{g l}}_{n}\right)$; see [4, Section 12] and [6, 8].

Let $\lambda=\left(\lambda_{1}, \ldots, \lambda_{n}\right)$ be an $n$-tuple of integers such that $\lambda_{1} \geqslant \cdots \geqslant \lambda_{n}$. The corresponding irreducible highest weight representation $L(\lambda)$ of $\mathrm{U}_{q}\left(\mathfrak{g l}_{n}\right)$ is generated by a nonzero vector $\xi$ such that

$$
\begin{aligned}
& \bar{t}_{i j} \xi=0 \quad \text { for } \quad 1 \leqslant i<j \leqslant n, \\
& t_{i i} \xi=q^{\lambda_{i}} \xi \quad \text { for } \quad 1 \leqslant i \leqslant n .
\end{aligned}
$$

This representation is a $q$-analogue of the irreducible $\mathfrak{g l}_{n}$-module with the highest weight $\lambda$. In particular, these modules have the same dimension.

We shall need an analogue of the Gelfand-Tsetlin basis for the module $L(\lambda)$ [12]; see also [13, Section 7.3.3] for more details. A pattern $\Lambda$ (associated with $\lambda$ ) is a sequence of rows of integers $\Lambda_{n}, \Lambda_{n-1}, \ldots, \Lambda_{1}$, where $\Lambda_{k}=\left(\lambda_{k 1}, \ldots, \lambda_{k k}\right)$ is the $k$-th row from the bottom, the top row $\Lambda_{n}$ coincides with $\lambda$, and the following betweenness conditions are satisfied: for $k=1, \ldots, n-1$

$$
\lambda_{k+1, i+1} \leqslant \lambda_{k i} \leqslant \lambda_{k+1, i} \quad \text { for } \quad i=1, \ldots, k .
$$

We shall be using the notation $l_{k i}=\lambda_{k i}-i+1$. Also, for any integer $m$ we set

$$
[m]=\frac{q^{m}-q^{-m}}{q-q^{-1}} .
$$

There exists a basis $\left\{\xi_{\Lambda}\right\}$ in $L(\lambda)$ parameterized by the patterns $\Lambda$ such that the action of the generators of $\mathrm{U}_{q}\left(\mathfrak{g l}_{n}\right)$ is given by

$$
\begin{aligned}
& t_{k k} \xi_{\Lambda}=q^{w_{k}} \xi_{\Lambda}, \quad w_{k}=\sum_{i=1}^{k} \lambda_{k i}-\sum_{i=1}^{k-1} \lambda_{k-1, i}, \\
& e_{k} \xi_{\Lambda}=-\sum_{j=1}^{k} \frac{\left[l_{k+1,1}-l_{k j}\right] \cdots\left[l_{k+1, k+1}-l_{k j}\right]}{\left[l_{k 1}-l_{k j}\right] \cdots \wedge_{j} \cdots\left[l_{k k}-l_{k j}\right]} \xi_{\Lambda+\delta_{k j}}, \\
& f_{k} \xi_{\Lambda}=\sum_{j=1}^{k} \frac{\left[l_{k-1,1}-l_{k j}\right] \cdots\left[l_{k-1, k-1}-l_{k j}\right]}{\left[l_{k 1}-l_{k j}\right] \cdots \wedge_{j} \cdots\left[l_{k k}-l_{k j}\right]} \xi_{\Lambda-\delta_{k j}},
\end{aligned}
$$

where $\Lambda \pm \delta_{k j}$ is obtained from $\Lambda$ by replacing the entry $\lambda_{k j}$ with $\lambda_{k j} \pm 1$, and $\xi_{\Lambda}$ is supposed to be equal to zero if $\Lambda$ is not a pattern; the symbol $\wedge_{j}$ indicates that the $j$-th factor is skipped.

The representation $L(\lambda)$ of $\mathrm{U}_{q}\left(\mathfrak{g l}_{n}\right)$ can be extended to a representation of $\mathrm{U}_{q}\left(\widehat{\mathfrak{g l}}_{n}\right)$ via the evaluation homomorphism; see (2.18). This $\mathrm{U}_{q}\left(\widehat{\mathfrak{g l}}_{n}\right)$-module is called the evaluation module. Its Drinfeld polynomials are given by

$$
P_{k}(u)=\left(1-q^{2 \lambda_{k+1}} u\right)\left(1-q^{2 \lambda_{k+1}+2} u\right) \cdots\left(1-q^{2 \lambda_{k}-2} u\right),
$$


for $k=1, \ldots, n-1$. We shall be using some formulas for the action of certain quantum minors of the matrices $T(u)$ and $\bar{T}(u)$ in the Gelfand-Tsetlin basis of $L(\lambda)$. Set

$$
T_{i j}(u)=\frac{u t_{i j}-u^{-1} \bar{t}_{i j}}{q-q^{-1}}
$$

Clearly, $\left(q-q^{-1}\right) T_{i j}(u)$ equals $u$ times the image of $t_{i j}\left(u^{2}\right)$ under the evaluation homomorphism (2.18). We also define the corresponding quantum minors $T_{b_{1} \cdots b_{r}}^{a_{1} \cdots a_{r}}(u)$ by using the formulas (2.26) or (2.28), so that, in particular, if $a_{1}<\cdots<a_{r}$ then

$$
T_{b_{1} \cdots b_{r}}^{a_{1} \cdots a_{r}}(u)=\sum_{\sigma \in \mathfrak{S}_{r}}(-q)^{-l(\sigma)} \cdot T_{a_{\sigma(1)} b_{1}}(u) \cdots T_{a_{\sigma(r)} b_{r}}\left(q^{-r+1} u\right)
$$

For any $k=1, \ldots, n$ we have in $L(\lambda)$,

$$
T_{1 \cdots k}^{1 \cdots k}(u) \xi_{\Lambda}=\prod_{i=1}^{k} \frac{u q^{l_{k i}}-u^{-1} q^{-l_{k i}}}{q-q^{-1}} \xi_{\Lambda} .
$$

Indeed, the coefficients of the polynomial $T_{1 \cdots k}^{1 \cdots k}(u)$ commute with the elements of the subalgebra $\mathrm{U}_{q}\left(\mathfrak{g l}_{k}\right)$ and so the relation is verified by the application of the polynomial to the highest vector of the $\mathrm{U}_{q}\left(\mathfrak{g l}_{k}\right)$-module $L\left(\Lambda_{k}\right)$. Furthermore, for any $k=1, \ldots, n-1$ and $j=1, \ldots, k$ we have

$$
T_{1 \cdots k-1, k+1}^{1 \cdots k}\left(q^{-l_{k j}}\right) \xi_{\Lambda}=-\left[l_{k+1,1}-l_{k j}\right] \cdots\left[l_{k+1, k+1}-l_{k j}\right] \xi_{\Lambda+\delta_{k j}}
$$

and

$$
T_{1 \cdots k}^{1 \cdots k-1, k+1}\left(q^{-l_{k j}}\right) \xi_{\Lambda}=\left[l_{k-1,1}-l_{k j}\right] \cdots\left[l_{k-1, k-1}-l_{k j}\right] \xi_{\Lambda-\delta_{k j}}
$$

The formulas (4.11) and (4.12) appeared for the first time in [25] in a slightly different form. For the proof of (4.11), it suffices to use the relation

$$
T_{1 \cdots k-1, k+1}^{1 \cdots k}(u)=T_{1 \cdots k}^{1 \cdots k}(u) e_{k}-q e_{k} T_{1 \cdots k}^{1 \cdots k}(u)
$$

which can be deduced from (2.19), and then apply (4.5) and (4.10). The proof of (4.12) is similar with the use of (4.6) and (4.10).

Suppose now that $m$ is a non-negative integer and let $\lambda=\left(\lambda_{1}, \ldots, \lambda_{m+n}\right)$ be an $(m+n)$-tuple of integers such that $\lambda_{1} \geqslant \cdots \geqslant \lambda_{m+n}$. Furthermore, let $\mu=\left(\mu_{1}, \ldots, \mu_{m}\right)$ be an $m$-tuple of integers such that $\mu_{1} \geqslant \cdots \geqslant \mu_{m}$. Regarding $\mathrm{U}_{q}\left(\mathfrak{g l}_{m}\right)$ as a natural subalgebra of $\mathrm{U}_{q}\left(\mathfrak{g l}_{m+n}\right)$, consider the space $\operatorname{Hom}_{\mathrm{U}_{q}\left(\mathfrak{g l}_{m}\right)}(L(\mu), L(\lambda))$. This vector space is isomorphic to the subspace $L(\lambda)_{\mu}^{+}$of $L(\lambda)$ which consists of $\mathrm{U}_{q}\left(\mathfrak{g l}_{m}\right)$-singular vectors of weight $\mu$,

$$
\begin{gathered}
L(\lambda)_{\mu}^{+}=\left\{\eta \in L(\lambda) \mid \bar{t}_{i j} \eta=0 \quad 1 \leqslant i<j \leqslant m \quad\right. \text { and } \\
\left.t_{i i} \eta=q^{\mu_{i}} \eta \quad i=1, \ldots, m\right\} .
\end{gathered}
$$

Note that $L(\lambda)_{\mu}^{+}$is nonzero if and only if

$$
\lambda_{i} \geqslant \mu_{i} \geqslant \lambda_{i+n} \quad \text { for } \quad i=1, \ldots, m
$$


see e.g. [4]. We shall assume that these inequalities hold. In that case, $L(\lambda)_{\mu}^{+}$admits a basis $\left\{\zeta_{\Lambda}\right\}$ labelled by the trapezium Gelfand-Tsetlin patterns $\Lambda$ of the form

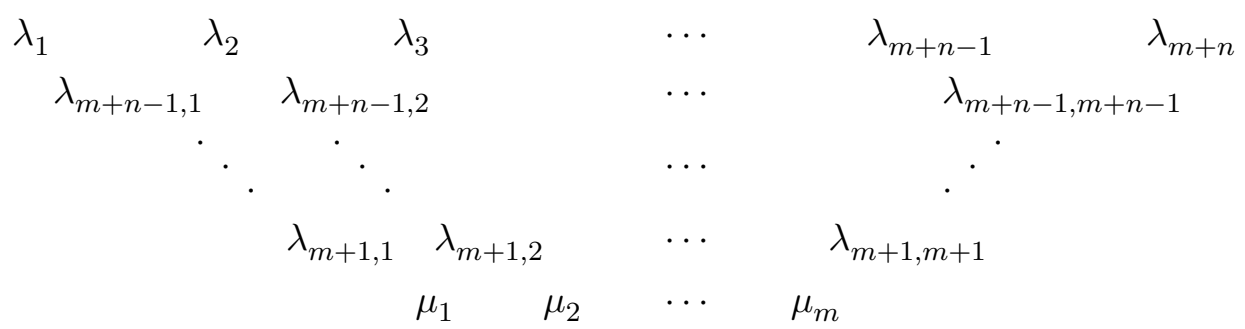

These arrays are formed by integers $\lambda_{k i}$ satisfying the betweenness conditions

$$
\lambda_{k+1, i+1} \leqslant \lambda_{k i} \leqslant \lambda_{k+1, i}
$$

for $k=m, m+1, \ldots, m+n-1$ and $1 \leqslant i \leqslant k$, where we have set

$$
\lambda_{m i}=\mu_{i}, \quad i=1, \ldots, m \quad \text { and } \quad \lambda_{m+n, j}=\lambda_{j}, \quad j=1, \ldots, m+n .
$$

Observe that $L(\lambda)_{\mu}^{+}$is a natural module over the subalgebra $\mathrm{U}_{q}^{\prime}\left(\mathfrak{g l}_{n}\right)$ of $\mathrm{U}_{q}\left(\mathfrak{g l}_{m+n}\right)$ generated by the elements $t_{i j}$ and $\bar{t}_{i j}$ with $m+1 \leqslant i, j \leqslant m+n$. Consider the trapezium pattern $\Lambda^{0}$ with the entries given by

$$
\lambda_{m+k, i}^{0}=\min \left\{\lambda_{i}, \mu_{i-k}\right\}, \quad k=1, \ldots, n-1, \quad i=1, \ldots, m+k,
$$

where we assume $\mu_{j}=+\infty$ if $j \leqslant 0$. Then

$$
t_{p p} \zeta_{\Lambda^{0}}=q^{w_{p}} \zeta_{\Lambda^{0}}, \quad w_{p}=\sum_{i=1}^{p} \lambda_{p i}^{0}-\sum_{i=1}^{p-1} \lambda_{p-1, i}^{0}, \quad p=m+1, \ldots, m+n
$$

The weight $\left(w_{m+1}, \ldots, w_{m+n}\right)$ of $\zeta_{\Lambda^{0}}$ is maximal with respect to the standard ordering on the set of weights of the $\mathrm{U}_{q}^{\prime}\left(\mathfrak{g l}_{n}\right)$-module $L(\lambda)_{\mu}^{+}$.

We now make $L(\lambda)_{\mu}^{+}$into a module over the quantum affine algebra $\mathrm{U}_{q}\left(\widehat{\mathfrak{g l}}_{n}\right)$ by taking the composition of the homomorphism (3.13) with the evaluation homomorphism $\mathrm{U}_{q}\left(\widehat{\mathfrak{g l}}_{m+n}\right) \rightarrow$ $\mathrm{U}_{q}\left(\mathfrak{g l}_{m+n}\right)$ given by (2.18). Thus, we can write the formulas for the action of $\mathrm{U}_{q}\left(\widehat{\mathfrak{g l}} \mathrm{l}_{n}\right)$ on $L(\lambda)_{\mu}^{+}$ in the form

$$
\begin{aligned}
& t_{i j}(u) \mapsto\left(\operatorname{qdet}\left(T-\bar{T} u^{-1}\right)_{\mathcal{A} \mathcal{A}}\right)^{-1} \cdot \operatorname{qdet}\left(T-\bar{T} u^{-1}\right)_{\mathcal{A}_{i} \mathcal{A}_{j}} \\
& \bar{t}_{i j}(u) \mapsto\left(\operatorname{qdet}(\bar{T}-T u)_{\mathcal{A \mathcal { A }}}\right)^{-1} \cdot \operatorname{qdet}(\bar{T}-T u)_{\mathcal{A}_{i} \mathcal{A}_{j}}
\end{aligned}
$$

where $\mathcal{A}=\{1, \ldots, m\}$ and $\mathcal{A}_{i}=\{1, \ldots, m, m+i\}$ while $T$ and $\bar{T}$ denote the generator matrices for the algebra $\mathrm{U}_{q}\left(\widehat{\mathfrak{g l}}_{m+n}\right)$. The subspace $L(\lambda)_{\mu}^{+}$of $L(\lambda)$ is preserved by these operators due to relations (2.32).

Theorem 4.1. The representation $L(\lambda)_{\mu}^{+}$of $\mathrm{U}_{q}\left(\widehat{\mathfrak{g l}}_{n}\right)$ is irreducible.

Proof. By Corollary 3.9, for the action of quantum minors on $L(\lambda)_{\mu}^{+}$we can write

$$
t_{b_{1} \cdots b_{r}}^{a_{1} \cdots a_{r}}\left(u^{2}\right) \mapsto T_{1 \cdots m}^{1 \cdots m}(u)^{-1} T_{1 \cdots m, m+b_{1} \cdots m+b_{r}}^{1 \cdots m}(u) \prod_{i=1}^{r} \frac{q-q^{-1}}{u q^{-m-i+1}},
$$


where $a_{i}, b_{i} \in\{1, \ldots, n\}$. Note that the coefficients of the polynomial $T_{1 \cdots m}^{1 \cdots m}(u)$ act on $L(\lambda)_{\mu}^{+}$ as scalar operators found from

$$
T_{1 \cdots m}^{1 \cdots m}(u) \zeta_{\Lambda}=\prod_{i=1}^{m} \frac{u q^{\ell_{i}}-u^{-1} q^{-\ell_{i}}}{q-q^{-1}} \zeta_{\Lambda}, \quad \ell_{i}=\mu_{i}-i+1 .
$$

On the other hand, by the formulas (4.11) and (4.12), each of the operators

$$
T_{1 \cdots m, m+1 \cdots m+k-1, m+k+1}^{1 \cdots m, m+1 \cdots m+k}\left(q^{-l_{m+k, i}}\right) \quad \text { and } \quad T_{1 \cdots m, m+1 \cdots m+k}^{1 \cdots m, m+1 \cdots m+k-1, m+k+1}\left(q^{-l_{m+k, i}}\right)
$$

takes a basis vector $\zeta_{\Lambda}$ of $L(\lambda)_{\mu}^{+}$to another basis vector with a nonzero coefficient provided the respective array $\Lambda+\delta_{m+k, i}$ or $\Lambda-\delta_{m+k, i}$ is a pattern.

Remark 2. The above proof actually shows that $L(\lambda)_{\mu}^{+}$is irreducible as a representation of the $q$-Yangian $\mathrm{Y}_{q}\left(\mathfrak{g l}_{n}\right)$.

Moreover, as the coefficients of the quantum minors

$$
t_{1 \cdots r}^{1 \cdots r}(u), \quad t_{1 \cdots r-1, r+1}^{1 \cdots r}(u), \quad t_{1 \cdots r}^{1 \cdots r-1, r+1}(u)
$$

and

$$
\bar{t}_{1 \cdots r}^{1 \cdots r}(u), \quad \bar{t}_{1 \cdots r-1, r+1}^{1 \cdots r}(u), \quad \bar{t}_{1 \cdots r}^{1 \cdots r-1, r+1}(u)
$$

with $r \geqslant 1$ generate the algebra $\mathrm{U}_{q}\left(\widehat{\mathfrak{g l}}_{n}\right)$, Corollary 3.9 together with the formulas $(4.10),(4.11)$ and (4.12) provide an explicit realization of the $\mathrm{U}_{q}\left(\widehat{\mathfrak{g l}}_{n}\right)$-module $L(\lambda)_{\mu}^{+}$.

Our next goal is to calculate the highest weight and Drinfeld polynomials of the $\mathrm{U}_{q}\left(\widehat{\mathfrak{g l}}_{n}\right)$ module $L(\lambda)_{\mu}^{+}$. As we have noticed above, $\zeta_{\Lambda^{0}}$ is a unique vector of maximal weight with respect to the standard ordering on the set of weights of the $\mathrm{U}_{q}^{\prime}\left(\mathfrak{g l}_{n}\right)$-module $L(\lambda)_{\mu}^{+}$. By the defining relations of $\mathrm{U}_{q}\left(\widehat{\mathfrak{g l}}_{n}\right)$, we have

$$
t_{j j}^{(0)} t_{i a}(u)=q^{\delta_{i j}-\delta_{j a}} t_{i a}(u) t_{j j}^{(0)} \quad \text { and } \quad t_{j j}^{(0)} \bar{t}_{i a}(u)=q^{\delta_{i j}-\delta_{j a}} \bar{t}_{i a}(u) t_{j j}^{(0)} .
$$

This implies that $\zeta_{\Lambda^{0}}$ is the highest vector of the $\mathrm{U}_{q}\left(\widehat{\mathfrak{g l}}_{n}\right)$-module $L(\lambda)_{\mu}^{+}$. The following theorem provides an identification of this module; cf. [19, 26]. Given three integers $i, j, k$ we shall denote by middle $\{i, j, k\}$ that of the three which is between the two others.

Theorem 4.2. The $\mathrm{U}_{q}\left(\widehat{\mathfrak{g l}}_{n}\right)$-module $L(\lambda)_{\mu}^{+}$is isomorphic to the highest weight representation $L(\nu(u), \bar{\nu}(u))$, where the components of the highest weight are found by

$$
\nu_{k}(u)=\frac{\left(q^{\nu_{k}^{(1)}}-q^{-\nu_{k}^{(1)}} u^{-1}\right) \cdots\left(q_{k}^{\nu_{k}^{(m+1)}}-q^{-\nu_{k}^{(m+1)}+2 m} u^{-1}\right)}{\left(q^{\mu_{1}}-q^{-\mu_{1}} u^{-1}\right) \cdots\left(q^{\mu_{m}}-q^{-\mu_{m}+2 m-2} u^{-1}\right)}
$$

and

$$
\bar{\nu}_{k}(u)=\frac{\left(q^{-\nu_{k}^{(1)}}-q^{\nu_{k}^{(1)}} u\right) \cdots\left(q^{-\nu_{k}^{(m+1)}}-q^{\nu_{k}^{(m+1)}}-2 m u\right)}{\left(q^{-\mu_{1}}-q^{\mu_{1}} u\right) \cdots\left(q^{-\mu_{m}}-q^{\mu_{m}-2 m+2} u\right)},
$$

where $k=1, \ldots, n$ and

$$
\nu_{k}^{(i)}=\operatorname{middle}\left\{\mu_{i-1}, \mu_{i}, \lambda_{k+i-1}\right\}
$$

assuming $\mu_{m+1}=-\infty$, and $\mu_{0}=+\infty$. 
Proof. Due to Theorem 4.1, we only need to calculate the highest weight of the $\mathrm{U}_{q}\left(\widehat{\mathfrak{g l}}_{n}\right)$-module $L(\lambda)_{\mu}^{+}$. As $\zeta_{\Lambda^{0}}$ is the highest vector, we have for any $1 \leqslant k \leqslant n$

$$
t_{1 \cdots k}^{1 \cdots k}(u) \zeta_{\Lambda^{0}}=\nu_{1}(u) \nu_{2}\left(q^{-2} u\right) \cdots \nu_{k}\left(q^{-2 k+2} u\right) \zeta_{\Lambda^{0}} .
$$

On the other hand, by Corollary 3.9, the action of this quantum minor can be found by applying the evaluation homomorphism (2.18) to the following series with coefficients in $\mathrm{U}_{q}\left(\widehat{\mathfrak{g l}}_{m+n}\right)$,

$$
\left(t_{1 \ldots m}^{1 \ldots m}(u)\right)^{-1} \cdot t_{1 \ldots m, m+1 \ldots m+k}^{1 \ldots m, m+1 \ldots m+k}(u),
$$

and then applying the image to the vector $\zeta_{\Lambda^{0}}$. This yields the relation

$$
\begin{aligned}
\nu_{1}(u) & \nu_{2}\left(q^{-2} u\right) \cdots \nu_{k}\left(q^{-2 k+2} u\right) \\
& =\frac{\left(q^{\lambda_{m+k, 1}^{(0)}}-q^{-\lambda_{m+k, 1}^{(0)}} u^{-1}\right) \cdots\left(q^{\lambda_{m+k, m+k}^{(0)}}-q^{-\lambda_{m+k, m+k}^{(0)}+2 m+2 k-2} u^{-1}\right)}{\left(q^{\mu_{1}}-q^{-\mu_{1}} u^{-1}\right) \cdots\left(q^{\mu_{m}}-q^{-\mu_{m}+2 m-2} u^{-1}\right)} .
\end{aligned}
$$

Hence, replacing here $k$ by $k-1$ and $u$ by $q^{2 k-2} u$, we get

$$
\nu_{k}(u)=\frac{\left(q^{\lambda_{m+k, 1}^{(0)}}-q^{-\lambda_{m+k, 1}^{(0)}-2 k+2} u^{-1}\right) \cdots\left(q^{\lambda_{m+k, m+k}^{(0)}}-q^{-\lambda_{m+k, m+k}^{(0)}+2 m} u^{-1}\right)}{\left(q^{\lambda_{m+k-1,1}^{(0)}}-q^{-\lambda_{m+k-1,1}^{(0)}-2 k+2} u^{-1}\right) \cdots\left(q^{\lambda_{m+k-1, m+k-1}^{(0)}}-q^{-\lambda_{m+k-1, m+k-1}^{(0)}+2 m-2} u^{-1}\right)} .
$$

By the definition (4.13) of the pattern $\Lambda^{0}$, we have

$$
\lambda_{m+k, i}^{(0)}=\lambda_{m+k-1, i}^{(0)}=\lambda_{i} \quad \text { for } \quad i=1, \ldots, k-1
$$

while for any $1 \leqslant j \leqslant m$ we have

$$
\begin{aligned}
& \frac{\left(q^{\lambda_{m+k, k+j-1}^{(0)}}-q^{-\lambda_{m+k, k+j-1}^{(0)}+2 j-2} u^{-1}\right)}{\left(q^{\lambda_{m+k-1, k+j-1}^{(0)}}-q^{-\lambda_{m+k-1, k+j-1}^{(0)}+2 j-2} u^{-1}\right)} \cdot\left(q^{\mu_{j}}-q^{-\mu_{j}+2 j-2} u^{-1}\right) \\
& \quad=\left(q^{\nu_{k}^{(j)}}-q^{-\nu_{k}^{(j)}+2 j-2} u^{-1}\right)
\end{aligned}
$$

completing the proof of the formula for $\nu_{k}(u)$.

Similarly, using Corollary 3.9 again, we find that

$$
\begin{aligned}
\bar{\nu}_{1}(u) & \bar{\nu}_{2}\left(q^{-2} u\right) \cdots \bar{\nu}_{k}\left(q^{-2 k+2} u\right) \\
= & \frac{\left(q^{-\lambda_{m+k, 1}^{(0)}}-q^{\lambda_{m+k, 1}^{(0)}} u\right) \cdots\left(q^{-\lambda_{m+k, m+k}^{(0)}}-q^{\lambda_{m+k, m+k}^{(0)}-2 m-2 k+2} u\right)}{\left(q^{-\mu_{1}}-q^{\mu_{1}} u\right) \cdots\left(q^{-\mu_{m}}-q^{\mu_{m}-2 m+2} u\right)},
\end{aligned}
$$

and then proceed in the same way as in the calculation of $\nu_{k}(u)$.

In the case where the components of $\lambda$ and $\mu$ are nonnegative we may regard them as partitions. Consider the corresponding skew diagram $\lambda / \mu$ and denote by $c(\alpha)$ the content of a box $\alpha \in \lambda / \mu$ so that $c(\alpha)=j-i$ if $\alpha$ occurs in row $i$ and column $j$.

Corollary 4.3. The Drinfeld polynomials $P_{1}(u), \ldots, P_{n-1}(u)$ corresponding to the $\mathrm{U}_{q}\left(\widehat{\mathfrak{g l}}_{n}\right)$ module $L(\lambda)_{\mu}^{+}$are given by

$$
P_{k}(u)=\prod_{i=1}^{m+1}\left(1-q^{2 \nu_{k+1}^{(i)}-2 i+2} u\right)\left(1-q^{2 \nu_{k+1}^{(i)}-2 i+4} u\right) \cdots\left(1-q^{2 \nu_{k}^{(i)}-2 i} u\right),
$$

for $k=1, \ldots, n-1$. If $\lambda$ and $\mu$ are partitions then the formula can also be written as

$$
P_{k}(u)=\prod_{\alpha}\left(1-q^{2 c(\alpha)} u\right),
$$

where $\alpha$ runs over the top boxes of the columns of height $k$ in the diagram of $\lambda / \mu$. 
Proof. The formulas follow from (4.1) and Theorem 4.2.

Note that if $m=0$ then $L(\lambda)_{\mu}^{+}$may be regarded as the evaluation module $L(\lambda)$ over $\mathrm{U}_{q}\left(\widehat{\mathfrak{g l}}_{n}\right)$. Clearly, the Drinfeld polynomials provided by Corollary 4.3 coincide with those given by (4.7).

Remark 3. The arguments of this section can be applied to produce simpler proofs of the Yangian analogues of Theorem 4.2 and Corollary 4.3; cf. [19, 26].

Finally, we calculate the Gelfand-Tsetlin characters of the skew representations. Following [3, Section 5.2], introduce the set $\mathcal{P}_{n}$ of all power series of the form $a(u)=a_{1}\left(u_{1}\right) \cdots a_{n}\left(u_{n}\right)$, where the $u_{1}, \ldots, u_{n}$ are indeterminates and each $a_{i}(u)$ is a power series in $u^{-1}$. We shall denote by $a_{i}^{(r)}$ the coefficient of $u^{-r}$ of this series. Consider the group algebra $\mathbb{Z}\left[\mathcal{P}_{n}\right]$ of the Abelian group $\mathcal{P}_{n}$ whose elements are finite linear combinations of the form $\sum m_{a(u)}[a(u)]$, where $m_{a(u)} \in \mathbb{Z}$.

We shall be working with the $q$-Yangian $\mathrm{Y}_{q}\left(\mathfrak{g l}_{n}\right)$. Let us introduce the series $h_{i}(u)$ with coefficients in $\mathrm{Y}_{q}\left(\mathfrak{g l}_{n}\right)$ by the formulas

$$
h_{i}(u)=t_{1 \ldots i-1}^{1 \ldots i-1}\left(q^{2 i-2} u\right)^{-1} t_{1 \ldots i}^{1 \ldots i}\left(q^{2 i-2} u\right), \quad i=1, \ldots, n .
$$

Due to $(2.32)$, the coefficients $h_{i}^{(r)}$ of all the series form a commutative subalgebra of $\mathrm{Y}_{q}\left(\mathfrak{g l}_{n}\right)$. Now, if $V$ is a finite-dimensional representation of $\mathrm{Y}_{q}\left(\mathfrak{g l}_{n}\right)$ and $a(u) \in \mathcal{P}_{n}$, the corresponding Gelfand-Tsetlin subspace $V_{a(u)}$ consists of the vectors $v \in V$ with the property that for each $i=1, \ldots, n$ and $r \geqslant 0$ there exists $p \geqslant 1$ such that $\left(h_{i}^{(r)}-a_{i}^{(r)}\right)^{p} v=0$. Then the Gelfand-Tsetlin character of $V$ is defined by

$$
\operatorname{ch} V=\sum_{a(u) \in \mathcal{P}_{n}}\left(\operatorname{dim} V_{a(u)}\right)[a(u)]
$$

cf. $[9,14]$. By analogy with [3, Section 6.2], introduce the following special elements of the group algebra $\mathbb{Z}\left[\mathcal{P}_{n}\right]$ by

$$
x_{i, a}=\left[\frac{q^{a+i}-q^{-a-i} u_{i}^{-1}}{q^{a+i-1}-q^{-a-i+1} u_{i}^{-1}}\right], \quad 1 \leqslant i \leqslant n, \quad a \in \mathbb{C} .
$$

We make $L(\lambda)_{\mu}^{+}$into a module over the $q$-Yangian $\mathrm{Y}_{q}\left(\mathfrak{g l}_{n}\right)$ by taking the composition of the homomorphism $\mathrm{Y}_{q}\left(\mathfrak{g l}_{n}\right) \rightarrow \mathrm{Y}_{q}\left(\mathfrak{g l}_{m+n}\right)$ given by the first relation in (3.13) with the evaluation homomorphism $\mathrm{Y}_{q}\left(\mathfrak{g l}_{m+n}\right) \rightarrow \mathrm{U}_{q}\left(\mathfrak{g l}_{m+n}\right)$ given by

$$
T(u) \mapsto \frac{T-\bar{T} u^{-1}}{1-u^{-1}} .
$$

This additional factor, as compared to (2.18), will ensure the character formulas look simpler. For the same purpose, we shall also assume that the components of $\lambda$ and $\mu$ are non-negative so that we can consider the corresponding skew diagram $\lambda / \mu$. The formulas in the general case will then be obtained by an obvious modification. A semistandard $\lambda / \mu$-tableau $\mathcal{T}$ is obtained by writing the elements of the set $\{1, \ldots, n\}$ into the boxes of the diagram of $\lambda / \mu$ in such a way that the elements in each row weakly increase while the elements in each column strictly increase. By $\mathcal{T}(\alpha)$ we denote the entry of $\mathcal{T}$ in the box $\alpha \in \lambda / \mu$.

Corollary 4.4. The Gelfand-Tsetlin character of the $\mathrm{Y}_{q}\left(\mathfrak{g l}_{n}\right)$-module $L(\lambda)_{\mu}^{+}$is given by

$$
\operatorname{ch} L(\lambda)_{\mu}^{+}=\sum_{\mathcal{T}} \prod_{\alpha \in \lambda / \mu} x_{\mathcal{T}(\alpha), c(\alpha)},
$$

summed over all semistandard $\lambda / \mu$-tableaux $\mathcal{T}$. 
Proof. First, we consider the particular case $m=0$ so that $L(\lambda)_{\mu}^{+}$coincides with the evaluation module $L(\lambda)$. The coefficients of the quantum determinant of $\mathrm{Y}_{q}\left(\mathfrak{g l}_{n}\right)$ act on $L(\lambda)$ as scalar operators found from

$$
\left.\operatorname{qdet} T(u)\right|_{L(\lambda)}=\prod_{i=1}^{n} \frac{q^{\lambda_{i}}-q^{-\lambda_{i}+2 i-2} u^{-1}}{1-q^{2 i-2} u^{-1}} .
$$

Observe that, regarding $\lambda$ as a diagram, we can write the product here as

$$
\prod_{\alpha \in \lambda} \frac{q^{c(\alpha)+1}-q^{-c(\alpha)-1} u^{-1}}{q^{c(\alpha)}-q^{-c(\alpha)} u^{-1}} .
$$

Note also that the quantum determinant can be factorized as

$$
q \operatorname{det} T(u)=h_{1}(u) h_{2}\left(q^{-2} u\right) \cdots h_{n}\left(q^{-2 n+2} u\right) .
$$

Now we employ the well-known bijection between the patterns associated with $\lambda$ and the semistandard $\lambda$-tableaux. Namely, the pattern $\Lambda$ can be viewed as the sequence of diagrams

$$
\Lambda_{1} \subseteq \Lambda_{2} \subseteq \cdots \subseteq \Lambda_{n}=\lambda
$$

where the $\Lambda_{k}$ are the rows of $\Lambda$. The betweenness conditions (4.2) mean that the skew diagram $\Lambda_{k} / \Lambda_{k-1}$ is a horizontal strip; see, e.g., Macdonald [18, Chapter 1]. The corresponding semistandard tableau is obtained by placing the entry $k$ into each box of $\Lambda_{k} / \Lambda_{k-1}$. By the above observation, for any basis vector $\xi_{\Lambda} \in L(\lambda)$ and any $1 \leqslant k \leqslant n$ we have

$$
h_{1}(u) h_{2}\left(q^{-2} u\right) \cdots h_{k}\left(q^{-2 k+2} u\right) \xi_{\Lambda}=\prod_{\alpha \in \Lambda_{k}} \frac{q^{c(\alpha)+1}-q^{-c(\alpha)-1} u^{-1}}{q^{c(\alpha)}-q^{-c(\alpha)} u^{-1}} \xi_{\Lambda},
$$

so that

$$
h_{k}\left(q^{-2 k+2} u\right) \xi_{\Lambda}=\prod_{\alpha \in \Lambda_{k} / \Lambda_{k-1}} \frac{q^{c(\alpha)+1}-q^{-c(\alpha)-1} u^{-1}}{q^{c(\alpha)}-q^{-c(\alpha)} u^{-1}} \xi_{\Lambda} .
$$

Thus, the element of $\mathbb{Z}\left[\mathcal{P}_{n}\right]$ corresponding to the eigenvalue of $h_{k}\left(u_{k}\right)$ on $\xi_{\Lambda}$ coincides with the product

$$
\prod_{\alpha \in \Lambda_{k} / \Lambda_{k-1}} x_{k, c(\alpha)}
$$

This proves the claim for the case $m=0$.

In the skew case $(m \geqslant 1)$ we use Corollary 3.9 which implies that formula (4.15) remains valid when the action is considered on the basis vector $\zeta_{\Lambda} \in L(\lambda)_{\mu}^{+}$instead of $\xi_{\Lambda}$. Here we denote by $\Lambda_{k}$ the row $\left(\lambda_{m+k, 1}, \ldots, \lambda_{m+k, m+k}\right)$ of the trapezium pattern $\Lambda$ and use a natural bijection between the trapezium patterns and the semistandard $\lambda / \mu$-tableaux.

Remark 4. The Gelfand-Tsetlin character of the corresponding skew module $L(\lambda)_{\mu}^{+}$over the Yangian $\mathrm{Y}\left(\mathfrak{g l}_{n}\right)$ is given by the same formula as in Corollary 4.4, where $x_{i, a}$ is now defined by $x_{i, a}=\left[1+\left(u_{i}+a+i-1\right)^{-1}\right]$; see [3, Section 6.2]. The skew representations of the Yangians and quantum affine algebras were studied in the literature from various viewpoints providing different interpretations of the character formula of Corollary 4.4; see e.g. [1, 5, 19, 23, 26]. For the evaluation modules, a calculation similar to the above can be found e.g. in [8, Section 4.5], [3, Section 7.4]. 
One can easily extend the definition of the Gelfand-Tsetlin character to representations of $\mathrm{U}_{q}\left(\widehat{\mathfrak{g l}}_{n}\right)$ by considering the commutative subalgebra generated by the coefficients of the series $h_{i}(u)$ together with the coefficients of the $\bar{h}_{i}(u)$ which are defined in the same way with the use of the quantum minors of $\bar{T}(u)$. However, by the results of [9], the eigenvalues of the $\bar{h}_{i}(u)$ on finite-dimensional representations are essentially determined by those of $h_{i}(u)$. In particular, the character formula for the $\mathrm{U}_{q}\left(\widehat{\mathfrak{g l}}_{n}\right)$-module $L(\lambda)_{\mu}^{+}$will have the form given in Corollary 4.4.

\section{Olshanski algebra associated with $\mathrm{U}_{q}\left(\mathfrak{g l}_{n}\right)$}

In this section we consider $q$ as a formal variable so that the quantum algebras are regarded as algebras over $\mathbb{C}(q)$. Denote by $\widetilde{\mathrm{U}}_{q}\left(\mathfrak{g l}_{n}\right)$ the subalgebra of $\mathrm{U}_{q}\left(\mathfrak{g l}_{n}\right)$ generated by the elements

$$
\tau_{i j}=t_{i j} \bar{t}_{j j}, \quad i>j \quad \text { and } \quad \bar{\tau}_{i j}=\bar{t}_{i j} \bar{t}_{j j}, \quad i \leqslant j .
$$

Since in the algebra $\mathrm{U}_{q}\left(\mathfrak{g l}_{n}\right)$ we have

$$
q^{\delta_{j a}} t_{i a} \bar{t}_{j j}=q^{\delta_{i j}} \bar{t}_{j j} t_{i a} \quad \text { and } \quad q^{\delta_{j a}} \bar{t}_{i a} \bar{t}_{j j}=q^{\delta_{i j}} \bar{t}_{j j} \bar{t}_{i a}
$$

we may regard $\widetilde{\mathrm{U}}_{q}\left(\mathfrak{g l}_{n}\right)$ as an associative algebra generated by the elements $\tau_{i j}$ with $i>j$ and $\bar{\tau}_{i j}$ with $i \leqslant j$ subject to the defining relations

$$
\begin{aligned}
& q^{\delta_{i j}+\delta_{j a}} \tau_{i a} \tau_{j b}-q^{\delta_{i b}+\delta_{a b}} \tau_{j b} \tau_{i a}=\left(q-q^{-1}\right) q^{\delta_{i a}}\left(\delta_{b<a}-\delta_{i<j}\right) \tau_{j a} \tau_{i b}, \\
& q^{\delta_{i j}+\delta_{j a}} \bar{\tau}_{i a} \bar{\tau}_{j b}-q^{\delta_{i b}+\delta_{a b}} \bar{\tau}_{j b} \bar{\tau}_{i a}=\left(q-q^{-1}\right) q^{\delta_{i a}}\left(\delta_{b<a}-\delta_{i<j}\right) \bar{\tau}_{j a} \bar{\tau}_{i b}, \\
& q^{\delta_{i j}+\delta_{j a}} \bar{\tau}_{i a} \tau_{j b}-q^{\delta_{i b}+\delta_{a b}} \tau_{j b} \bar{\tau}_{i a}=\left(q-q^{-1}\right) q^{\delta_{i a}}\left(\delta_{b<a} \tau_{j a} \bar{\tau}_{i b}-\delta_{i<j} \bar{\tau}_{j a} \tau_{i b}\right),
\end{aligned}
$$

where

$$
\tau_{i j}=\bar{\tau}_{j i}=0, \quad 1 \leqslant i<j \leqslant n, \quad \text { and } \quad \tau_{i i}=1, \quad i=1, \ldots, n .
$$

For any positive integer $n$ the algebra $\widetilde{\mathrm{U}}_{q}\left(\mathfrak{g l}_{n-1}\right)$ can be identified with a subalgebra of $\widetilde{\mathrm{U}}_{q}\left(\mathfrak{g l}_{n}\right)$ generated by the elements $\tau_{i j}$ and $\bar{\tau}_{i j}$ with $1 \leqslant i, j \leqslant n-1$.

Fix a nonnegative integer $m$ such that $m \leqslant n$ and denote by $\widetilde{\mathrm{U}}_{q}\left(\mathfrak{g l}_{n, m}\right)$ the subalgebra of $\widetilde{\mathrm{U}}_{q}\left(\mathfrak{g l}_{n}\right)$ generated by the elements $\tau_{i j}$ and $\bar{\tau}_{i j}$ with $m+1 \leqslant i, j \leqslant n$. This subalgebra is isomorphic to $\widetilde{\mathrm{U}}_{q}\left(\mathfrak{g l}_{n-m}\right)$. Let $\mathrm{A}_{m}(n)$ denote the centralizer of $\widetilde{\mathrm{U}}_{q}\left(\mathfrak{g l}_{n, m}\right)$ in $\widetilde{\mathrm{U}}_{q}\left(\mathfrak{g l}_{n}\right)$. Also, let $\mathrm{A}(n)^{0}$ denote the centralizer of the element $\bar{\tau}_{n n}$ in $\widetilde{\mathrm{U}}_{q}\left(\mathfrak{g l} \mathfrak{l}_{n}\right)$ and let $\mathrm{I}(n)$ be the left ideal in $\widetilde{\mathrm{U}}_{q}\left(\mathfrak{g l}_{n}\right)$ generated by the elements $\bar{\tau}_{i n}, i=1, \ldots, n$. Then the Poincaré-Birkhoff-Witt theorem for the algebra $\mathrm{U}_{q}\left(\mathfrak{g l}_{n}\right)$ implies that $\mathrm{I}(n)^{0}=\mathrm{I}(n) \cap \mathrm{A}(n)^{0}$ is a two-sided ideal in $\mathrm{A}(n)^{0}$ and one has a vector space decomposition

$$
\mathrm{A}(n)^{0}=\mathrm{I}(n)^{0} \oplus \widetilde{\mathrm{U}}_{q}\left(\mathfrak{g l}_{n-1}\right) .
$$

Therefore, the projection of $\mathrm{A}(n)^{0}$ onto $\widetilde{\mathrm{U}}_{q}\left(\mathfrak{g l}_{n-1}\right)$ with the kernel $\mathrm{I}(n)^{0}$ is an algebra homomorphism. If $m<n$ then its restriction to the subalgebra $\mathrm{A}_{m}(n)$ defines a homomorphism

$$
o_{n}: \mathrm{A}_{m}(n) \rightarrow \mathrm{A}_{m}(n-1)
$$

Note that the algebra $\mathrm{A}_{m}(n)$ inherits the filtration of $\widetilde{\mathrm{U}}_{q}\left(\mathfrak{g l}_{n}\right)$ defined by

$$
\operatorname{deg} \tau_{i j}=0, \quad i>j, \quad \text { and } \quad \operatorname{deg} \bar{\tau}_{i j}=1, \quad i \leqslant j .
$$

Clearly, the homomorphism $o_{n}$ is filtration preserving. 
Definition 5.1. The Olshanski algebra $\mathrm{A}_{m}$ is defined as the projective limit of the sequence of the algebras $\mathrm{A}_{m}(n), n \geqslant m$, with respect to the homomorphisms

$$
\mathrm{A}_{m}(m) \stackrel{o_{m+1}}{\longleftarrow} \mathrm{A}_{m}(m+1) \stackrel{o_{m+2}}{\longleftarrow} \cdots \stackrel{o_{n}}{\longleftarrow} \mathrm{A}_{m}(n) \stackrel{o_{n+1}}{\longleftarrow} \cdots
$$

where the limit is taken in the category of filtered associative algebras.

An element of the algebra $\mathrm{A}_{m}$ is a sequence of the form $a=\left(a_{m}, a_{m+1}, \ldots, a_{n}, \ldots\right)$ with $a_{n} \in \mathrm{A}_{m}(n), o_{n}\left(a_{n}\right)=a_{n-1}$ for $n>m$, and

$$
\operatorname{deg} a=\sup _{n \geqslant m} \operatorname{deg} a_{n}<\infty,
$$

where deg $a_{n}$ denotes the degree of $a_{n}$ in $\widetilde{\mathrm{U}}_{q}\left(\mathfrak{g l}_{n}\right)$. If $b=\left(b_{m}, b_{m+1}, \ldots, b_{n}, \ldots\right)$ is another element of $\mathrm{A}_{m}$ then the product $a b$ is the sequence

$$
a b=\left(a_{m} b_{m}, a_{m+1} b_{m+1}, \ldots, a_{n} b_{n}, \ldots\right) .
$$

We define the algebra $\widetilde{\mathrm{U}}_{q}\left(\mathfrak{g l} \mathrm{l}_{\infty}\right)$ as the inductive limit of the algebras $\widetilde{\mathrm{U}}_{q}\left(\mathfrak{g l} \mathrm{l}_{n}\right)$ with respect to the natural embeddings $\widetilde{\mathrm{U}}_{q}\left(\mathfrak{g l}_{n}\right) \hookrightarrow \widetilde{\mathrm{U}}_{q}\left(\mathfrak{g l}_{n+1}\right)$,

$$
\widetilde{\mathrm{U}}_{q}\left(\mathfrak{g l}_{\infty}\right)=\bigcup_{n \geqslant 1} \widetilde{\mathrm{U}}_{q}\left(\mathfrak{g l}_{n}\right)
$$

Note that the algebra $\mathrm{A}_{0}(n)$ coincides with the center of $\widetilde{\mathrm{U}}_{q}\left(\mathfrak{g l}_{n}\right)$. The elements of the algebra $\mathrm{A}_{0}$ can therefore be regarded as virtual Casimir elements for the algebra $\widetilde{\mathrm{U}}_{q}\left(\mathfrak{g l}_{\infty}\right)$.

Let us apply the evaluation homomorphism

$$
\mathrm{Y}_{q}\left(\mathfrak{g l}_{n}\right) \rightarrow \mathrm{U}_{q}\left(\mathfrak{g l}_{n}\right), \quad T(u) \mapsto T-\bar{T} u^{-1},
$$

see $(2.18)$, to the quantum determinant qdet $T(u)$. We get the polynomial in $u^{-1}$,

$$
\begin{aligned}
\operatorname{qdet}\left(T-\bar{T} u^{-1}\right)= & \sum_{\sigma \in \mathfrak{S}_{n}}(-q)^{-l(\sigma)}\left(t_{\sigma(1) 1}-\bar{t}_{\sigma(1) 1} u^{-1}\right) \\
& \times\left(t_{\sigma(2) 2}-\bar{t}_{\sigma(2) 2} q^{2} u^{-1}\right) \cdots\left(t_{\sigma(n) n}-\bar{t}_{\sigma(n) n} q^{2 n-2} u^{-1}\right),
\end{aligned}
$$

whose coefficients are central in $\mathrm{U}_{q}\left(\mathfrak{g l}_{n}\right)$. Applying the Harish-Chandra homomorphism (2.11) to the coefficients (see Section 2), we find

$$
\chi: \operatorname{qdet}\left(T-\bar{T} u^{-1}\right) \mapsto q^{n(n-1) / 2} \cdot\left(x_{1}-x_{1}^{-1} u^{-1}\right) \cdots\left(x_{n}-x_{n}^{-1} u^{-1}\right) .
$$

Hence, the coefficients of the polynomial (5.5) generate the center $\mathrm{Z}_{q}$ of $\mathrm{U}_{q}\left(\mathfrak{g l}_{n}\right)$.

The product

$$
d_{n}(u)=\operatorname{qdet}\left(T-\bar{T} u^{-1}\right) \cdot \bar{t}_{11} \cdots \bar{t}_{n n}
$$

is a polynomial in $u^{-1}$ with constant term 1 whose coefficients belong to the center of the subalgebra $\widetilde{\mathrm{U}}_{q}\left(\mathfrak{g l}_{n}\right)$. Explicitly, this polynomial can be written as

$$
d_{n}(u)=\sum_{\sigma \in \mathfrak{S}_{n}}(-q)^{-l(\sigma)} q^{\operatorname{ind}(\sigma)}\left(\tau_{\sigma(1) 1}-\bar{\tau}_{\sigma(1) 1} u^{-1}\right) \cdots\left(\tau_{\sigma(n) n}-\bar{\tau}_{\sigma(n) n} q^{2 n-2} u^{-1}\right),
$$

where $\operatorname{ind}(\sigma)=\sharp\{i=1, \ldots, n \mid \sigma(i)<i\}$. Write

$$
d_{n}(u)=1+d_{n}^{(1)} u^{-1}+\cdots+d_{n}^{(n)} u^{-n} .
$$


Proposition 5.2. The elements $d_{n}^{(1)}, \ldots, d_{n}^{(n)}$ are algebraically independent and generate the center of the algebra $\widetilde{\mathrm{U}}_{q}\left(\mathfrak{g l}_{n}\right)$.

Proof. Any central element $z$ of $\widetilde{\mathrm{U}}_{q}\left(\mathfrak{g l}_{n}\right)$ must commute with $\bar{\tau}_{11}, \ldots, \bar{\tau}_{n n}$. Using the isomorphism (2.10), and writing $z$ as a linear combination of the corresponding basis monomials, we conclude that $z$ must also commute with $t_{11}, \ldots, t_{n n}$. Therefore, $z$ belongs to the center $\mathrm{Z}_{q}$ of $\mathrm{U}_{q}\left(\mathfrak{g l}_{n}\right)$. Hence, $z$ is a polynomial in $t_{0}, t_{0}^{-1}, d_{n}^{(1)}, \ldots, d_{n}^{(n)}$, where $t_{0}=t_{11} \cdots t_{n n}$. However, such a polynomial does not belong to the subalgebra $\widetilde{\mathrm{U}}_{q}\left(\mathfrak{g l}_{n}\right)$ unless it only contains non-positive even powers of $t_{0}$. Since $t_{0}^{-2}$ coincides with $d_{n}^{(n)}$ up to a nonzero constant factor, this proves that the center of $\widetilde{\mathrm{U}}_{q}\left(\mathfrak{g l}_{n}\right)$ is generated by $d_{n}^{(1)}, \ldots, d_{n}^{(n)}$.

Finally, the image of $d_{n}(u)$ under the Harish-Chandra homomorphism is given by

$$
\chi: d_{n}(u) \mapsto\left(1-x_{1}^{-2} u^{-1}\right) \cdots\left(1-x_{n}^{-2} u^{-1}\right),
$$

thus proving the algebraic independence of $d_{n}^{(1)}, \ldots, d_{n}^{(n)}$.

Applying the homomorphism (5.2) with $m=0$ to the coefficients of the polynomial $d_{n}(u)$ we find immediately that

$$
o_{n}: d_{n}(u) \mapsto d_{n-1}(u)
$$

Since the degree of the element $d_{n}^{(k)}$ does not exceed $k$ for all $n \geqslant k$, we may define a virtual Casimir element $d^{(k)} \in \mathrm{A}_{0}$ for any $k \geqslant 1$ as the sequence

$$
d^{(k)}=\left(d_{n}^{(k)} \mid n \geqslant 0\right),
$$

where we set $d_{n}^{(k)}=0$ for $n<k$. In order to get an alternative expression for the virtual Casimir elements $d^{(k)}$, denote by $\mathfrak{S}_{\infty}$ the group of finite permutations of the set of positive integers, so that for any $p \in \mathfrak{S}_{\infty}$ we have $p(l)=l$ for all sufficiently large $l$. Define the virtual quantum determinant as the formal power series

$$
d(u)=1+d^{(1)} u^{-1}+d^{(2)} u^{-2}+\cdots .
$$

Using the expression

$$
d(u)=\sum_{\sigma \in \mathfrak{S}_{\infty}}(-q)^{-l(\sigma)} q^{\operatorname{ind}(\sigma)}\left(\tau_{\sigma(1) 1}-\bar{\tau}_{\sigma(1) 1} u^{-1}\right)\left(\tau_{\sigma(2) 2}-\bar{\tau}_{\sigma(2) 2} q^{2} u^{-1}\right) \cdots,
$$

we can regard the coefficients $d^{(k)}$ as certain formal series of elements of $\widetilde{\mathrm{U}}_{q}\left(\mathfrak{g l}_{\infty}\right)$.

The following description of the algebra $A_{0}$ is implied by Proposition 5.2.

Proposition 5.3. The elements $d^{(1)}, d^{(2)}, \ldots$ are algebraically independent and generate the algebra $\mathrm{A}_{0}$.

We are now in a position to establish a relationship between the Olshanski algebra $A_{m}$ and the $q$-Yangian $\mathrm{Y}_{q}\left(\mathfrak{g l}_{m}\right)$. It will be convenient to work with the subalgebra $\widetilde{\mathrm{Y}}_{q}\left(\mathfrak{g l}_{m}\right)$ of $\mathrm{Y}_{q}\left(\mathfrak{g l}_{m}\right)$ generated by the coefficients of the series

$$
\tau_{i j}(u)=t_{i j}(u) \bar{t}_{j j}^{(0)}, \quad 1 \leqslant i, j \leqslant m .
$$

As with the algebra $\widetilde{\mathrm{U}}_{q}\left(\mathfrak{g l}_{m}\right)$, using the relations

$$
q^{\delta_{j a}} t_{i a}(u) \bar{t}_{j j}^{(0)}=q^{\delta_{i j}} \bar{t}_{j j}^{(0)} t_{i a}(u) \quad \text { and } \quad q^{\delta_{j a}} \bar{t}_{i a}(u) \bar{t}_{j j}^{(0)}=q^{\delta_{i j}} \bar{t}_{j j}^{(0)} \bar{t}_{i a}(u),
$$


it is easy to write down the defining relations of $\widetilde{Y}_{q}\left(\mathfrak{g l}_{m}\right)$ in terms of the coefficients $\tau_{i j}^{(r)}$ of the series $\tau_{i j}(u)$.

Now we use the quantum Sylvester theorem (Theorem 3.6). Taking the composition of the homomorphism (3.8) and the evaluation homomorphism (5.4), we obtain a homomorphism $\mathrm{Y}_{q}\left(\mathfrak{g l}_{m}\right) \rightarrow \mathrm{U}_{q}\left(\mathfrak{g l}_{n}\right)$ which can be written as

$$
t_{i j}(u) \mapsto \operatorname{qdet}\left(T-\bar{T} u^{-1}\right)_{\mathcal{B}_{i} \mathcal{B}_{j}},
$$

where we use the quantum determinant of the submatrix of $T-\bar{T} u^{-1}$ corresponding to the rows $\mathcal{B}_{i}$ and columns $\mathcal{B}_{j}$, where $\mathcal{B}_{i}$ denotes the set $\{i, m+1, \ldots, n\}$. By the relations (2.32), the image of the series $t_{i j}(u)$ under the homomorphism (5.9) commutes with the elements $t_{k l}$ and $\bar{t}_{k l}$ with $m+1 \leqslant k, l \leqslant n$. Introduce the matrices $\mathcal{T}=\left[\tau_{i j}\right]$ and $\overline{\mathcal{T}}=\left[\bar{\tau}_{i j}\right]$ with $1 \leqslant i, j \leqslant n$ and set

$$
\operatorname{qdet}^{\prime}\left(\mathcal{T}-\overline{\mathcal{T}} u^{-1}\right)_{\mathcal{B}_{i} \mathcal{B}_{j}}=\operatorname{qdet}\left(T-\bar{T} u^{-1}\right)_{\mathcal{B}_{i} \mathcal{B}_{j}} \cdot \bar{t}_{j j} \bar{t}_{m+1, m+1} \cdots \bar{t}_{n n}
$$

An explicit formula for this quantum minor in terms of $\tau_{i j}$ and $\bar{\tau}_{i j}$ can be written with the use of the parameter $\operatorname{ind}(\sigma)$ as in (5.6). Since the product $\bar{t}_{m+1, m+1} \ldots \bar{t}_{n n}$ commutes with $\operatorname{qdet}\left(T-\bar{T} u^{-1}\right)_{\mathcal{B}_{i} \mathcal{B}_{j}} \cdot \bar{t}_{j j}$ (see $\left.(2.32)\right)$, the mapping

$$
\varphi_{n}: \tau_{i j}(u) \mapsto \operatorname{qdet}^{\prime}\left(\mathcal{T}-\overline{\mathcal{T}} u^{-1}\right)_{\mathcal{B}_{i} \mathcal{B}_{j}}
$$

defines a homomorphism $\widetilde{\mathrm{Y}}_{q}\left(\mathfrak{g l}_{m}\right) \rightarrow \widetilde{\mathrm{U}}_{q}\left(\mathfrak{g l}_{n}\right)$. Furthermore, the same relation (2.32) implies that the image of the homomorphism (5.10) is contained in the centralizer $\mathrm{A}_{m}(n)$ so that we have a homomorphism

$$
\varphi_{n}: \widetilde{\mathrm{Y}}_{q}\left(\mathfrak{g l}_{m}\right) \rightarrow \mathrm{A}_{m}(n) .
$$

Using the explicit formula for $\operatorname{qdet}^{\prime}\left(\mathcal{T}-\overline{\mathcal{T}} u^{-1}\right)_{\mathcal{B}_{i} \mathcal{B}_{j}}$ as in (5.6), we find that the diagram

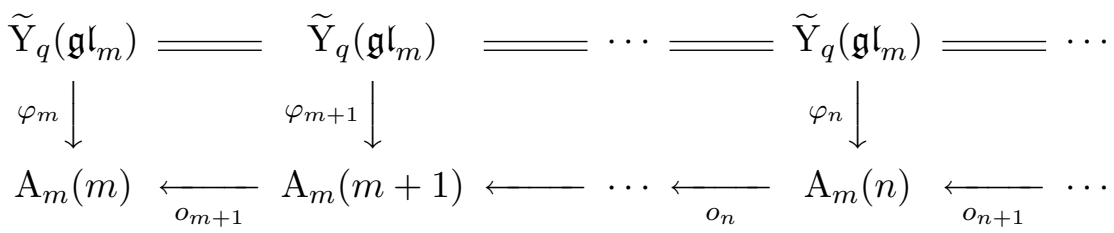

is commutative. Note that the image of the generator $\tau_{i j}^{(r)}$ of $\widetilde{Y}_{q}\left(\mathfrak{g l}_{m}\right)$ under $\varphi_{n}$ has degree $\leqslant r$ for any $n$. Hence the sequence of homomorphisms $\left(\varphi_{n} \mid n \geqslant m\right)$ defines an algebra homomorphism $\varphi: \widetilde{\mathrm{Y}}_{q}\left(\mathfrak{g l}_{m}\right) \rightarrow \mathrm{A}_{m}$ which can be written in terms of the virtual quantum determinants by

$$
\varphi: \tau_{i j}(u) \mapsto \operatorname{qdet}^{\prime}\left(\mathcal{T}-\overline{\mathcal{T}} u^{-1}\right)_{\mathcal{B}_{i} \mathcal{B}_{j}}
$$

where $\mathcal{B}_{i}$ now denotes the infinite set $\{i, m+1, m+2, \ldots\}$.

Theorem 5.4. The homomorphism $\varphi$ is an algebra embedding of $\widetilde{Y}_{q}\left(\mathfrak{g l}_{m}\right)$ into the algebra $\mathrm{A}_{m}$.

Proof. We shall use a weak form of the Poincaré-Birkhoff-Witt theorem for the algebra $\widetilde{Y}_{q}\left(\mathfrak{g l}_{m}\right)$ which can be verified by a direct argument; see e.g. [22, Lemma 3.2] where a similar result is proved for a twisted version of the $q$-Yangian. By this theorem, the ordered monomials in the generators $\tau_{i j}^{(r)}$ span the algebra $\tilde{Y}_{q}\left(\mathfrak{g l}_{m}\right)$. It will be sufficient to prove that the images of these monomials under the homomorphism $\varphi$ are linearly independent.

As in Section 2, set $\mathcal{A}=\mathbb{C}\left[q, q^{-1}\right]$ and consider the $\mathcal{A}$-subalgebra $\widetilde{\mathrm{U}}_{\mathcal{A}}$ of $\widetilde{\mathrm{U}}_{q}\left(\mathfrak{g l}_{n}\right)$ generated by the elements $\tau_{i j}$ and $\bar{\tau}_{i j}$. Denote by $\mathcal{P}_{n}$ the algebra of polynomials in independent variables 
$x_{i j}$ with $1 \leqslant i, j \leqslant n$. By the Poincaré-Birkhoff-Witt theorem for the algebra $\mathrm{U}_{q}\left(\mathfrak{g l}_{n}\right)$, we have an isomorphism

$$
\widetilde{\mathrm{U}}_{\mathcal{A}} \otimes_{\mathcal{A}} \mathbb{C} \cong \mathcal{P}_{n}
$$

given by

$$
\tau_{i j} \mapsto x_{i j} \quad \text { for } \quad i>j \quad \text { and } \quad \bar{\tau}_{i j} \mapsto x_{i j} \quad \text { for } \quad i \leqslant j
$$

where the action of $\mathcal{A}$ on $\mathbb{C}$ is defined via the evaluation $q=1$.

Suppose now that the image under $\varphi$ of a nontrivial linear combination of the ordered monomials in the generators $\tau_{i j}^{(r)}$ is zero. We may assume that all coefficients of this linear combination belong to $\mathcal{A}$. Moreover, we may also assume that at least one coefficient does not vanish at $q=1$ so that the image of this linear combination under the isomorphism (5.11) yields a nontrivial linear combination of the corresponding elements of $\mathcal{P}_{n}$. Observe that the image of the quantum determinant $\operatorname{qdet}^{\prime}\left(\mathcal{T}-\overline{\mathcal{T}} u^{-1}\right)_{\mathcal{B}_{i} \mathcal{B}_{j}}$ occurring in (5.10) under the isomorphism (5.11) coincides with the usual determinant $\operatorname{det}\left(X-\bar{X} u^{-1}\right)_{\mathcal{B}_{i} \mathcal{B}_{j}}$, where $X$ denote the lower triangular matrix with entries $x_{i j}$ for $i>j$ and with all diagonal entries equal to 1 while $\bar{X}$ is the upper triangular matrix with entries $x_{i j}$ for $i \leqslant j$. Write

$$
\operatorname{det}\left(X-\bar{X} u^{-1}\right)_{\mathcal{B}_{i} \mathcal{B}_{j}}=\lambda_{i j}^{(0)}-\lambda_{i j}^{(1)} u^{-1}+\cdots+(-1)^{n-m+1} \lambda_{i j}^{(n-m+1)} u^{-n+m-1} .
$$

The proof will be completed if we show that given any positive integer $p$, there exists a sufficiently large value of $n$ such that the polynomials $\lambda_{i j}^{(r)}$ with $0 \leqslant r \leqslant p$ and $1 \leqslant i, j \leqslant m$ (assuming $i>j$ for $r=0$ ) are algebraically independent. This will lead to a contradiction with the assumption that the ordered monomials in the $\tau_{i j}^{(r)}$ are linearly dependent.

We shall be proving that the corresponding map

$$
\Lambda_{n}: \mathbb{C}^{n^{2}} \rightarrow \mathbb{C}^{p m^{2}+m(m-1) / 2},
$$

given by

$$
\left(x_{i j} \mid 1 \leqslant i, j \leqslant n\right) \mapsto\left(\lambda_{k l}^{(r)} \mid 1 \leqslant k, l \leqslant m, \quad 0 \leqslant r \leqslant p\right)
$$

with $k>l$ for $r=0$, is surjective. We need two auxiliary lemmas. For any $r \geqslant 1$ we let $e_{r}\left(z_{1}, \ldots, z_{l}\right)$ denote the $r$-th elementary symmetric function in variables $z_{1}, \ldots, z_{l}$ with $l \geqslant r$ so that

$$
e_{r}\left(z_{1}, \ldots, z_{l}\right)=\sum_{1 \leqslant i_{1}<\cdots<i_{r} \leqslant l} z_{i_{1}} \cdots z_{i_{r}} .
$$

We also set $e_{0}\left(z_{1}, \ldots, z_{l}\right)=1$ and $e_{r}\left(z_{1}, \ldots, z_{l}\right)=0$ for $r<0$. Let $l$ be a positive integer and let $\alpha_{1}, \ldots, \alpha_{l}$ be distinct complex parameters. For each $i=1, \ldots, l$ set

$$
e_{r i}=e_{r}\left(\alpha_{1}, \ldots, \widehat{\alpha}_{i}, \ldots, \alpha_{l}\right) \text {, }
$$

where the hat indicates the symbol to be omitted. For any $1 \leqslant p \leqslant l$ consider the matrix

$$
\mathcal{E}=\left(\begin{array}{ccc}
e_{01} & \cdots & e_{0 l} \\
e_{11} & \cdots & e_{1 l} \\
\vdots & \ddots & \vdots \\
e_{p-1,1} & \cdots & e_{p-1, l}
\end{array}\right)
$$


Lemma 5.5. All $p \times p$ minors of the matrix $\mathcal{E}$ are nonzero.

Proof. Introduce the polynomials $Q_{1}(t), \ldots, Q_{l}(t)$ by

$$
Q_{i}(t)=e_{0 i} t^{l-1}+e_{1 i} t^{l-2}+\cdots+e_{l-1, i}=\prod_{k=1, k \neq i}^{l}\left(t+\alpha_{k}\right) .
$$

They are linearly independent for if $c_{1} Q_{1}(t)+\cdots+c_{l} Q_{l}(t)=0$ then taking $t=-\alpha_{i}$ gives $c_{i}=0$ since the parameters $\alpha_{i}$ are all distinct.

Suppose now that columns $i_{1}, \ldots, i_{p}$ with $i_{1}<\cdots<i_{p}$ of the matrix $\mathcal{E}$ are linearly dependent. This implies that a certain nontrivial linear combination $Q(t)=d_{1} Q_{i_{1}}(t)+\cdots+d_{p} Q_{i_{p}}(t)$ is a polynomial in $t$ of degree not exceeding $l-p-1$. However, $Q(t)$ has $l-p$ distinct roots $t=-\alpha_{j}$ with $j \in\{1, \ldots, l\} \backslash\left\{i_{1}, \ldots, i_{p}\right\}$, and so $Q(t)=0$. This contradicts the linear independence of the polynomials $Q_{i}(t)$.

Suppose now that $\mathcal{E}_{0}, \ldots, \mathcal{E}_{m-1}$ are given non-singular $p \times p$ matrices with complex entries.

Lemma 5.6. For any distinct complex numbers $\beta_{1}, \ldots, \beta_{m}$ the block matrix

$$
\left(\begin{array}{cccc}
\mathcal{E}_{0} & \beta_{1} \mathcal{E}_{1} & \cdots & \beta_{1}^{m-1} \mathcal{E}_{m-1} \\
\mathcal{E}_{0} & \beta_{2} \mathcal{E}_{1} & \cdots & \beta_{2}^{m-1} \mathcal{E}_{m-1} \\
\vdots & \vdots & \ddots & \vdots \\
\mathcal{E}_{0} & \beta_{m} \mathcal{E}_{1} & \cdots & \beta_{m}^{m-1} \mathcal{E}_{m-1}
\end{array}\right)
$$

is non-singular.

Proof. Suppose that a certain linear combination of the rows of the matrix with coefficients $c_{1}, c_{2}, \ldots, c_{p m}$ is zero. Since the rows of each of the matrices $\mathcal{E}_{j}$ are linearly independent, for any index $1 \leqslant i \leqslant p$ we have the linear relations

$$
\beta_{1}^{k} c_{i}+\beta_{2}^{k} c_{p+i}+\cdots+\beta_{m}^{k} c_{(m-1) p+i}=0, \quad k=0,1, \ldots, m-1 .
$$

As the $\beta_{j}$ are distinct, for each $i$ the system has only the trivial solution $c_{i}=c_{p+i}=\cdots=$ $c_{(m-1) p+i}=0$.

Now we shall show that the map (5.12) is surjective for any $n \geqslant(p+1) m$. In fact, we show that this map is surjective even when the variables $x_{k l}$ with $m+1 \leqslant k \leqslant n$ are specialized in the following way:

$$
x_{k l}=\beta_{k l}, \quad \text { for } \quad l=1, \ldots, m
$$

and

$$
x_{k l}=\delta_{k l} \alpha_{k}, \quad \text { for } \quad l=m+1, \ldots, n
$$

where $\alpha_{m+1}, \ldots, \alpha_{n}$ are distinct complex numbers and the $\beta_{k l}$ are certain complex numbers to be chosen below. Under this specialization, for each fixed value of the index $i \in\{1, \ldots, m\}$ consider the following restriction of the map (5.12)

$$
\Lambda_{n}^{(i)}: \mathbb{C}^{n} \rightarrow \mathbb{C}^{p m+i-1},
$$

given by

$$
\left(x_{i 1}, x_{i 2}, \ldots, x_{i n}\right) \mapsto\left(\lambda_{i 1}^{(0)}, \ldots, \lambda_{i 1}^{(p)}, \ldots, \lambda_{i, i-1}^{(0)}, \ldots, \lambda_{i, i-1}^{(p)}, \lambda_{i i}^{(1)}, \ldots, \lambda_{i i}^{(p)}, \ldots, \lambda_{i m}^{(1)}, \ldots, \lambda_{i m}^{(p)}\right),
$$


so that the functions $\lambda_{i j}^{(r)}$ are defined by the expansions

$$
\left|\begin{array}{ccccc}
t x_{i j} & x_{i, m+1} & x_{i, m+2} & \cdots & x_{i n} \\
t \beta_{m+1, j} & t+\alpha_{m+1} & 0 & \cdots & 0 \\
t \beta_{m+2, j} & 0 & t+\alpha_{m+2} & \cdots & 0 \\
\vdots & \vdots & \vdots & \ddots & \vdots \\
t \beta_{n, j} & 0 & 0 & \cdots & t+\alpha_{n}
\end{array}\right|=t^{n-m+1} \lambda_{i j}^{(0)}+\cdots+t \lambda_{i j}^{(n-m)}
$$

for $j=1, \ldots, i-1$,

$$
\left|\begin{array}{ccccc}
t+x_{i i} & x_{i, m+1} & x_{i, m+2} & \cdots & x_{i n} \\
t \beta_{m+1, i} & t+\alpha_{m+1} & 0 & \cdots & 0 \\
t \beta_{m+2, i} & 0 & t+\alpha_{m+2} & \cdots & 0 \\
\vdots & \vdots & \vdots & \ddots & \vdots \\
t \beta_{n, i} & 0 & 0 & \cdots & t+\alpha_{n}
\end{array}\right|=t^{n-m+1}+t^{n-m} \lambda_{i i}^{(1)}+\cdots+\lambda_{i i}^{(n-m+1)}
$$

and

$$
\left|\begin{array}{ccccc}
x_{i j} & x_{i, m+1} & x_{i, m+2} & \cdots & x_{i n} \\
t \beta_{m+1, j} & t+\alpha_{m+1} & 0 & \cdots & 0 \\
t \beta_{m+2, j} & 0 & t+\alpha_{m+2} & \cdots & 0 \\
\vdots & \vdots & \vdots & \ddots & \vdots \\
t \beta_{n, j} & 0 & 0 & \cdots & t+\alpha_{n}
\end{array}\right|=t^{n-m} \lambda_{i j}^{(1)}+\cdots+\lambda_{i j}^{(n-m+1)},
$$

for $j=i+1, \ldots, m$. More explicitly, we have

$$
\lambda_{i j}^{(r)}=x_{i j} e_{r}\left(\alpha_{m+1}, \ldots, \alpha_{n}\right)-\sum_{k=m+1}^{n} \beta_{k j} x_{i k} e_{r-1}\left(\alpha_{m+1}, \ldots, \widehat{\alpha}_{k}, \ldots, \alpha_{n}\right)
$$

for $j=1, \ldots, i-1$ and $r \geqslant 0$,

$$
\begin{aligned}
\lambda_{i i}^{(r)}= & x_{i i} e_{r-1}\left(\alpha_{m+1}, \ldots, \alpha_{n}\right) \\
& -\sum_{k=m+1}^{n} \beta_{k i} x_{i k} e_{r-1}\left(\alpha_{m+1}, \ldots, \widehat{\alpha}_{k}, \ldots, \alpha_{n}\right)+e_{r}\left(\alpha_{m+1}, \ldots, \alpha_{n}\right)
\end{aligned}
$$

for $r \geqslant 1$, and

$$
\lambda_{i j}^{(r)}=x_{i j} e_{r-1}\left(\alpha_{m+1}, \ldots, \alpha_{n}\right)-\sum_{k=m+1}^{n} \beta_{k j} x_{i k} e_{r-1}\left(\alpha_{m+1}, \ldots, \widehat{\alpha}_{k}, \ldots, \alpha_{n}\right)
$$

for $j=i+1, \ldots, m$ and $r \geqslant 1$. Since the $\lambda_{i j}^{(r)}$ are linear functions in the variables $x_{i 1}, \ldots, x_{i n}$, in order to establish the surjectivity of the map (5.14), it will be sufficient to demonstrate that the rank of the corresponding coefficient matrix is maximal, that is, equal to $p m+i-1$. Writing down the matrix in an explicit form and using the observation that $\lambda_{i j}^{(0)}=x_{i j}$ for $j=1, \ldots, i-1$ 
we conclude that the claim will follow by proving that the matrix

$$
\left(\begin{array}{ccc}
-\beta_{m+1,1} e_{0, m+1} & \cdots & -\beta_{n 1} e_{0, n} \\
-\beta_{m+1,1} e_{1, m+1} & \cdots & -\beta_{n 1} e_{1, n} \\
\vdots & \cdots & \vdots \\
-\beta_{m+1,1} e_{p-1, m+1} & \cdots & -\beta_{n 1} e_{p-1, n} \\
\vdots & \cdots & \vdots \\
\vdots & \cdots & \vdots \\
-\beta_{m+1, m} e_{0, m+1} & \cdots & -\beta_{n m} e_{0, n} \\
-\beta_{m+1, m} e_{1, m+1} & \cdots & -\beta_{n m} e_{1, n} \\
\vdots & \cdots & \vdots \\
-\beta_{m+1, m} e_{p-1, m+1} & \cdots & -\beta_{n m} e_{p-1, n}
\end{array}\right)
$$

has rank $p m$, where $e_{r k}=e_{r}\left(\alpha_{m+1}, \ldots, \widehat{\alpha}_{k}, \ldots, \alpha_{n}\right)$. Now, we use the assumption $n-m \geqslant p m$ and Lemma 5.5. Giving appropriate values to the parameters $\beta_{k l}$, we may choose a submatrix of (5.15) of size $p m \times p m$ of the form (5.13), where all the $p \times p$ blocks $\mathcal{E}_{i}$ are non-singular and the parameters $\beta_{i}$ are distinct. Thus, the claim follows by the application of Lemma 5.6.

Finally, by allowing the index $i$ in (5.14) to vary and by choosing the parameters $\beta_{k l}$ in the same way for each value of $i \in\{1, \ldots, m\}$, we get a surjective map

$$
\mathbb{C}^{m n} \rightarrow \mathbb{C}^{p m^{2}+m(m-1) / 2},
$$

given by

$$
\left(x_{i j} \mid 1 \leqslant i \leqslant m, \quad 1 \leqslant j \leqslant n\right) \mapsto\left(\lambda_{i l}^{(r)} \mid 1 \leqslant i, l \leqslant m, \quad 0 \leqslant r \leqslant p\right)
$$

with $i>l$ for $r=0$, thus completing the proof.

The argument used in the proof of Theorem 5.4 provides a proof of the Poincaré-BirkhoffWitt theorem for the algebra $\widetilde{\mathrm{Y}}_{q}\left(\mathfrak{g l}_{m}\right)$ and hence for $\mathrm{Y}_{q}\left(\mathfrak{g l}_{m}\right)$.

Corollary 5.7. Given any ordering on the set of generators of the algebra $\mathrm{Y}_{q}\left(\mathfrak{g l}_{m}\right)$, the ordered monomials in the generators form a basis of $\mathrm{Y}_{q}\left(\mathfrak{g l}_{m}\right)$.

Proof. As we showed in the proof of Theorem 5.4, given any finite family of ordered monomials in the generators $\tau_{i j}^{(r)}$ of the algebra $\widetilde{\mathrm{Y}}_{q}\left(\mathfrak{g l}_{m}\right)$, we can choose a sufficiently large value of $n$ such that the images of the monomials under the homomorphism (5.10) are linearly independent. As the ordered monomials span $\widetilde{\mathrm{Y}}_{q}\left(\mathfrak{g l}_{m}\right)$, we may conclude that they form a basis of $\widetilde{\mathrm{Y}}_{q}\left(\mathfrak{g l}_{m}\right)$. The corresponding statement for the algebra $\mathrm{Y}_{q}\left(\mathfrak{g l}_{m}\right)$ is now immediate from the relations (5.7) and (5.8).

We believe an analogue of the tensor product decomposition (1.3) for the algebra $A_{m}$ takes place. Namely, denote by $\widetilde{\mathrm{A}}_{0}$ the commutative subalgebra of $\mathrm{A}_{m}$ generated by the coefficients of the virtual quantum determinant $\operatorname{qdet}^{\prime}\left(\mathcal{T}-\overline{\mathcal{T}} u^{-1}\right)_{\mathcal{B B}}$ with $\mathcal{B}=\{m+1, m+2, \ldots\}$.

Conjecture 5.8. We have the tensor product decomposition

$$
\mathrm{A}_{m}=\widetilde{\mathrm{A}}_{0} \otimes \tilde{\mathrm{Y}}_{q}\left(\mathfrak{g l}_{m}\right),
$$

where the Yangian $\widetilde{\mathrm{Y}}_{q}\left(\mathfrak{g l}_{m}\right)$ is identified with its image under the embedding $\varphi$.

Remark 5. A slight modification of the proof of Theorem 5.4 yields an alternative method to prove that the Yangian $\mathrm{Y}\left(\mathfrak{g l}_{m}\right)$ is embedded into the Olshanski algebra $\mathrm{A}_{m}$; cf. [19, 28]. Moreover, as with the $q$-Yangian, this also provides a new proof of the Poincaré-Birkhoff-Witt theorem for the Yangian $\mathrm{Y}\left(\mathfrak{g l}_{m}\right)$; cf. [2, 20, 24]. 


\section{Acknowledgements}

We would like to thank Boris Feigin for discussions and Serge Ovsienko for help with the proof of Theorem 5.4 .

[1] Bazhanov V.V., Reshetikhin N., Restricted solid-on-solid models connected with simply laced algebras and conformal field theory, J. Phys. A: Math. Gen., 1990, V.23, 1477-1492.

[2] Brundan J., Kleshchev A., Parabolic presentations of the Yangian $\mathrm{Y}\left(\mathfrak{g l}_{n}\right)$, Comm. Math. Phys., 2005, V.254, 191-220, math.QA/0407011.

[3] Brundan J., Kleshchev A., Representations of shifted Yangians and finite $W$-algebras, math.RT/0508003.

[4] Chari V., Pressley A., A guide to quantum groups, Cambridge University Press, 1994.

[5] Cherednik I.V., A new interpretation of Gelfand-Tzetlin bases, Duke Math. J., 1987, V.54, 563-577.

[6] Ding J., Frenkel I., Isomorphism of two realizations of quantum affine algebra U $(\widehat{g l}(n))$, Comm. Math. Phys., 1993, V.156, 277-300.

[7] Drinfeld V.G., Hopf algebras and the quantum Yang-Baxter equation, Soviet Math. Dokl., 1985, V.32, $254-258$.

[8] Frenkel E., Mukhin E., The Hopf algebra Rep $U_{q} \widehat{\mathfrak{g l}}_{\infty}$, Selecta Math., 2002, V.8, 537-635, math.QA/0103126.

[9] Frenkel E., Reshetikhin N., The $q$-characters of representations of quantum affine algebras and deformations of $\mathcal{W}$-algebras, Contemp. Math., 1999, V.248, 163-205, math.QA/9810055.

[10] Gelfand I.M., Retakh V.S., Determinants of matrices over noncommutative rings, Funct. Anal. Appl., 1991, V.25, 91-102.

[11] Jimbo M., A $q$-analogue of $U_{q}(\mathfrak{g l}(N+1))$, Hecke algebra and the Yang-Baxter equation, Lett. Math. Phys., 1986, V.11, 247-252.

[12] Jimbo M., Quantum R-matrix for the generalized Toda system, Comm. Math. Phys., 1986, V.102, 537-547.

[13] Klimyk A., Schmüdgen K., Quantum groups and their representations, Berlin, Springer, 1997.

[14] Knight H., Spectra of tensor products of finite-dimensional representations of Yangians, J. Algebra, 1995, V.174, 187-196.

[15] Krob D., Leclerc B., Minor identities for quasi-determinants and quantum determinants, Comm. Math. Phys., 1995, V.169, 1-23, hep-th/9411194.

[16] Kuznetsov V.B., Koornwinder T.H., Gauss hypergeometric function and quadratic R-matrix algebras, St. Petersburg Math. J., 1995, V.6, 595-618, hep-th/9311152.

[17] Kuznetsov V.B., ${ }_{3} F_{2}(1)$ hypergeometric function and quadratic $R$-matrix algebra, in Symmetries and Integrability of Difference Equations (Estérel, PQ, 1994), CRM Proc. Lecture Notes, Vol. 9, Providence, RI, Amer. Math. Soc., 1996, 185-197, math.QA/9411225.

[18] Macdonald I.G., Symmetric functions and Hall polynomials, Oxford, Oxford University Press, 1995.

[19] Molev A.I., Yangians and transvector algebras, Discrete Math., 2002, V.246, 231-253, math.QA/9811115.

[20] Molev A., Nazarov M., Olshanski G., Yangians and classical Lie algebras, Russian Math. Surveys, 1996, V.51. N 2, 205-282, hep-th/9409025.

[21] Molev A., Olshanski G., Centralizer construction for twisted Yangians, Selecta Math., 2000, V.6, 269-317, q-alg/9712050.

[22] Molev A., Ragoucy E., Sorba P., Coideal subalgebras in quantum affine algebras, Rev. Math. Phys., 2003, V.15, 789-822, math.QA/0208140.

[23] Nakai W., Nakanishi T., Paths, tableaux and $q$-characters of quantum affine algebras: the $C_{n}$ case, math.QA/0502041.

[24] Nazarov M., Yangian of the queer Lie superalgebra, Comm. Math. Phys., 1999, V.208, 195-223, math.QA/9902146.

[25] Nazarov M., Tarasov V., Yangians and Gelfand-Zetlin bases, Publ. Res. Inst. Math. Sci. Kyoto Univ., 1994, V.30, 459-478, hep-th/9302102.

[26] Nazarov M., Tarasov V., Representations of Yangians with Gelfand-Zetlin bases, J. Reine Angew. Math., 1998, V.496, 181-212, q-alg/9502008.

[27] Olshanski G.I., Extension of the algebra $U(g)$ for infinite-dimensional classical Lie algebras $g$, and the Yangians $Y(g l(m))$, Soviet Math. Dokl., 1988, V.36, 569-573. 
[28] Olshanski G.I., Representations of infinite-dimensional classical groups, limits of enveloping algebras, and Yangians, in Topics in Representation Theory, Editor A.A. Kirillov, Advances in Soviet Math., Vol. 2, Providence, RI, Amer. Math. Soc., 1991, 1-66.

[29] Olshanski G.I., Twisted Yangians and infinite-dimensional classical Lie algebras, in Quantum Groups, Editor P.P. Kulish, Lecture Notes in Math., Vol. 1510, Berlin-Heidelberg, Springer, 1992, 103-120.

[30] Reshetikhin N.Yu., Takhtajan L.A., Faddeev L.D., Quantization of Lie groups and Lie algebras, Leningrad Math. J., 1990, V.1, 193-225. 